\title{
A Random Field Formulation of Hooke's Law in All Elasticity Classes
}

\author{
Anatoliy Malyarenko ${ }^{1}$ (D) Martin Ostoja-Starzewski ${ }^{2}$
}

Received: 1 March 2016/ Published online: 7 December 2016

(C) The Author(s) 2016. This article is published with open access at Springerlink.com

\begin{abstract}
For each of the 8 symmetry classes of elastic materials, we consider a homogeneous random field taking values in the fixed point set $\mathrm{V}$ of the corresponding class, that is isotropic with respect to the natural orthogonal representation of a group lying between the isotropy group of the class and its normaliser. We find the general form of the correlation tensors of orders 1 and 2 of such a field, and the field's spectral expansion.
\end{abstract}

Keywords Elasticity class $\cdot$ Random field $\cdot$ Spectral expansion

Mathematics Subject Classification $60 \mathrm{G} 60 \cdot 74 \mathrm{~A} 40$

\section{Introduction}

Microstructural randomness is present in just about all solid materials. When dominant (macroscopic) length scales are large relative to microscales, one can safely work with deterministic homogeneous continuum models. However, when the separation of scales does not hold and spatial randomness needs to be accounted for, various concepts of continuum mechanics need to be re-examined and new methods developed. This involves: (1) being able to theoretically model and simulate any such randomness, and (2) using such results as input into stochastic field equations. In this paper, we work in the setting of linear elastic random media that are statistically wide-sense homogeneous and isotropic.

Regarding the modeling motivation (1), two basic issues are considered in this study: (i) type of anisotropy, and (ii) type of correlation structure. Now, with reference to Fig. 1

This material is based upon the research partially supported by the NSF under grants CMMI-1462749 and IP-1362146 (I/UCRC on Novel High Voltage/Temperature Materials and Structures).

A. Malyarenko

anatoliy.malyarenko@mdh.se

M. Ostoja-Starzewski

martinos@illinois.edu

1 Mälardalen University, Västerås, Sweden

2 University of Illinois at Urbana-Champaign, Urbana, IL, USA 


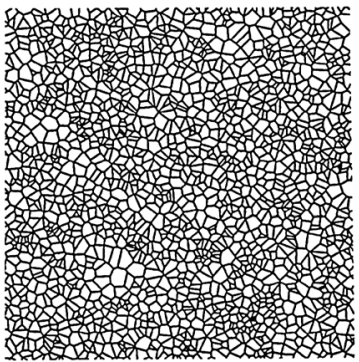

(a)

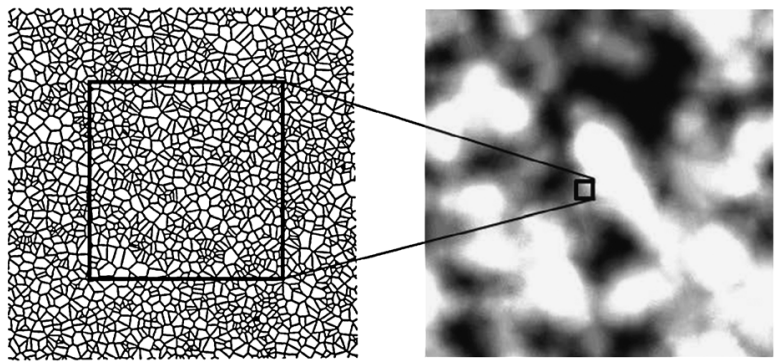

(b) (c)

Fig. 1 (a) A realisation of a Voronoi tesselation (or mosaic); (b) placing a mesoscale window leads, via upscaling, to a mesoscale random continuum approximation in (c)

showing a planar Voronoi tessellation of $E^{2}$ which serves as a planar geometric model of a polycrystal (although the same arguments apply in $E^{3}$ ), each cell may be occupied by a differently oriented crystal, with all the crystals belonging to any specific crystal class. The latter include:

- transverse isotropy modelling, say, sedimentary rocks at long wavelengths;

- tetragonal modelling, say, wulfenite $\left(\mathrm{PbMoO}_{4}\right)$;

- trigonal modelling, say, dolomite $\left(\mathrm{CaMg}\left(\mathrm{CO}_{3}\right)_{2}\right)$;

- orthotropic, modelling, say, wood;

- orthotropic modelling, say, orthoclase feldspar;

- triclinic, modelling, say, microcline feldspar.

Thus, we need to be able to model 4th-rank tensor random fields, pointwise taking values in any crystal class. While the crystal orientations from grain to grain are random, they are not spatially independent of each other - the assignment of crystal properties over the tessellation is not a white noise. This is precisely where the two-point characterisation of the random field of elasticity tensor is needed. While the simplest correlation structure to admit would be white-noise, a (much) more realistic model would account for any mathematically admissible correlation structures as dictated by the statistically wide-sense homogeneous and isotropic assumption. A specific correlation can then be fitted to physical measurements.

Regarding the modeling motivation (1), it may also be of interest to work with a mesoscale random continuum approximation defined by placing a mesoscale window at any spatial position as shown in Fig. 1(b). Clearly, the larger is the mesoscale window, the weaker are the random fluctuations in the mesoscale elasticity tensor: this is the trend to homogenise the material when upscaling from a statistical volume element (SVE) to a representative volume element (RVE), e.g., [27,30]. A simple paradigm of this upscaling, albeit only in terms of a scalar random field, is the opacity of a sheet of paper held against light: the further away is the sheet from our eyes, the more homogeneous it appears. Similarly, in the case of upscaling of elastic properties (which are tensor in character), on any finite scale there is (almost surely) an anisotropy, and this anisotropy, with mesoscale increasing, tends to zero hand-in-hand with the fluctuations. It is in the infinite mesoscale limit (i.e., RVE) that material isotropy is obtained as a consequence of the statistical isotropy.

Regarding the motivation (2) of this study, i.e., input of elasticity random fields into stochastic field equations, there are two principal routes: stochastic partial differential equations (SPDE) and stochastic finite elements (SFE). The classical paradigm of SPDE [19] can 
be written in terms of the anti-plane elastostatics (with $u \equiv u_{3}$ ):

$$
\nabla \cdot(C(\mathbf{x}, \omega) \nabla u)=0, \quad \mathbf{x} \in E^{2}, \omega \in \Omega
$$

with $C(\cdot, \omega)$ being spatial realisations of a scalar RF. In view of the foregoing discussion, (1) is well justified for a piecewise-constant description of realisations of a random medium such as a multiphase composite made of locally isotropic grains. However, in the case of a boundary value problem set up on coarser (i.e., mesoscales) scales, having continuous realisations of properties, a 2nd-rank tensor random field (TRF) of material properties would be much more appropriate, Fig. 1(c). The field equation should then read

$$
\nabla \cdot(\mathbf{C}(\mathbf{x}, \omega) \cdot \nabla u)=0, \quad \mathbf{x} \in E^{2}, \omega \in \Omega
$$

where $\mathrm{C}$ is the 2nd-rank tensor random field. Indeed, this type of upscaling is sorely needed in the stochastic finite element (SFE) method, where, instead of assuming the local isotropy of the elasticity tensor for each and every material volume (and, hence, the finite element), full triclinic-type anisotropy is needed [28].

Moving to the in-plane or $3 \mathrm{~d}$ elasticity, if one assumes local material isotropy, a simple way to introduce material spatial randomness is to take the pair of Lamé constants $(\lambda, \mu)$ as a "vector" random field, resulting in

$$
\mu \nabla^{2} \mathbf{u}+(\lambda+\mu) \nabla(\nabla \cdot \mathbf{u})+\nabla \mu \cdot\left(\nabla \boldsymbol{u}+(\nabla \mathbf{u})^{\top}\right)+\nabla \lambda \nabla \cdot \mathbf{u}=\rho \ddot{\mathbf{u}}
$$

as a generalisation of the classical Navier equation. However, just like in (1) above, the local isotropy is a crude approximation in view of micromechanics upscaling arguments, and (3) should be replaced by

$$
\nabla \cdot(\mathrm{C} \cdot \nabla \mathbf{u})=\rho \ddot{\mathbf{u}},
$$

where the stiffness $\mathrm{C}\left(=C_{i j k l} \mathbf{e}_{i} \otimes \mathbf{e}_{j} \otimes \mathbf{e}_{k} \otimes \mathbf{e}_{l}\right)$ is a TRF. At any scale finitely larger than the microstructural scale, it is almost surely (a.s.) anisotropic, e.g. [27, 29]. Clearly, instead of (3), one should work with this SPDE (4) for $\mathbf{u}$.

While the mathematical theory of SPDEs with anisotropic realisations is not yet developed, one powerful way to numerically solve such equations is through stochastic finite elements (SFE). However, the SFE, just like the SPDE, require a general representation of the random field $\mathrm{C}$, so it can be fitted to micromechanics upscaling studies, as well as its spectral expansion. Observe that each and every material volume (and, hence, the finite element) is an SVE of Fig. 1(c), so that a full triclinic-type anisotropy is needed: all the entries of the 4th-rank stiffness tensor $\mathrm{C}$ are non-zero with probability one. While a micromechanically consistent procedure for upscaling has been discussed in [33] and references cited there, general forms of the correlation tensors are sorely needed.

In this paper we develop second-order TRF models of linear hyperelastic media in each of the eight elasticity classes. That is, for each class, the fourth-rank elasticity tensor is taken as an isotropic and homogeneous random field in a three-dimensional Euclidean space, for which the one-point (mean) and two-point correlation functions need to be explicitly specified. The simplest case is that of an isotropic class, which implies that two Lamé constants are random fields. Next, we develop representations of seven higher crystal classes: cubic, transversely isotropic, trigonal, tetragonal, orthotropic, monoclinic, and triclinic. We also find the general form of field's spectral expansion for each of the eight isotropy classes. 


\section{The Formulation of the Problem}

Let $E=E^{3}$ be a three-dimensional Euclidean point space, and let $V$ be the translation space of $E$ with an inner product $(\cdot, \cdot)$. Following [35], the elements $A$ of $E$ are called the places in $E$. The symbol $B-A$ is the vector in $V$ that translates $A$ into $B$.

Let $\mathscr{B} \subset E$ be a deformable body. The strain tensor $\varepsilon(A), A \in \mathscr{B}$, is a configuration variable taking values in the symmetric tensor square $S^{2}(V)$ of dimension 6. Following [25], we call this space a state tensor space.

The stress tensor $\sigma(A)$ also takes values in $\mathrm{S}^{2}(V)$. This is a source variable, it describes the source of a field [34].

We work with materials obeying Hooke's law linking the configuration variable $\varepsilon(A)$ with the source variable $\sigma(A)$ by

$$
\sigma(A)=\mathrm{C}(A) \varepsilon(A), \quad A \in \mathscr{B} .
$$

Here the elastic modulus $\mathrm{C}$ is a linear map $\mathrm{C}(A): \mathrm{S}^{2}(V) \rightarrow \mathrm{S}^{2}(V)$. In linearised hyperelasticity, the map $\mathrm{C}(A)$ is symmetric, i.e., an element of a constitutive tensor space $\mathrm{V}=\mathrm{S}^{2}\left(\mathrm{~S}^{2}(V)\right)$ of dimension 21 .

We assume that $\mathrm{C}(A)$ is a single realisation of a random field. In other words, denote by $\mathfrak{B}(\mathrm{V})$ the $\sigma$-field of Borel subsets of $\mathrm{V}$. There is a probability space $(\Omega, \mathfrak{F}, \mathrm{P})$ and a mapping $\mathrm{C}: \mathscr{B} \times \Omega \rightarrow \mathrm{V}$ such that for any $A_{0} \in \mathscr{B}$ the mapping $\mathrm{C}\left(A_{0}, \omega\right): \Omega \rightarrow \mathrm{V}$ is $(\mathfrak{F}, \mathfrak{B}(\mathrm{V}))$-measurable.

Translate the whole body $\mathscr{B}$ by a vector $\mathbf{x} \in V$. The random fields $\mathrm{C}(A+\mathbf{x})$ and $\mathrm{C}(A)$ have the same finite-dimensional distributions. It is therefore convenient to assume that there is a random field defined on all of $E$ such that its restriction to $\mathscr{B}$ is equal to $\mathrm{C}(A)$. For brevity, denote the new field by the same symbol $\mathrm{C}(A)$ (but this time $A \in E$ ). The random field $\mathrm{C}(A)$ is strictly homogeneous, that is, the random fields $\mathrm{C}(A+\mathbf{x})$ and $\mathrm{C}(A)$ have the same finite-dimensional distributions. In other words, for each positive integer $n$, for each $\mathbf{x} \in V$, and for all distinct places $A_{1}, \ldots, A_{n} \in E$ the random elements $\mathrm{C}\left(A_{1}\right) \oplus \cdots \oplus \mathrm{C}\left(A_{n}\right)$ and $\mathrm{C}\left(A_{1}+\mathbf{x}\right) \oplus \cdots \oplus \mathrm{C}\left(A_{n}+\mathbf{x}\right)$ of the direct sum on $n$ copies of the space $\mathrm{V}$ have the same probability distribution.

Let $K$ be the material symmetry group of the body $\mathscr{B}$ acting in $V$. The group $K$ is a subgroup of the orthogonal group $\mathrm{O}(V)$. Fix a place $O \in \mathscr{B}$ and identify $E$ with $V$ by the map $f$ that maps $A \in E$ to $A-O \in V$. Then $K$ acts in $E$ and rotates the body $\mathscr{B}$ by

$$
g \cdot A=f^{-1} g f A, \quad g \in K, A \in \mathscr{B} .
$$

Let $A_{0} \in \mathscr{B}$. Under the above action of $K$ the point $A_{0}$ becomes $g \cdot A_{0}$. The random tensor $\mathrm{C}\left(A_{0}\right)$ becomes $\mathrm{S}^{2}\left(\mathrm{~S}^{2}(g)\right) \mathrm{C}\left(A_{0}\right)$. The random fields $\mathrm{C}(g \cdot A)$ and $\mathrm{S}^{2}\left(\mathrm{~S}^{2}(g)\right) \mathrm{C}(A)$ must have the same finite-dimensional distributions, because $g \cdot A_{0}$ is the same material point in a different place. Note that this property does not depend on a particular choice of the place $O$, because the field is strictly homogeneous.

To formalise the non-formal considerations of the above paragraph, note that the map $g \mapsto \mathrm{S}^{2}\left(\mathrm{~S}^{2}(g)\right)$ is an orthogonal representation of the group $K$, that is, a continuous map from $K$ to the orthogonal group $\mathrm{O}(\mathrm{V})$ that respects the group operations:

$$
\mathrm{S}^{2}\left(\mathrm{~S}^{2}\left(g_{1} g_{2}\right)\right)=\mathrm{S}^{2}\left(\mathrm{~S}^{2}\left(g_{1}\right)\right) \mathrm{S}^{2}\left(\mathrm{~S}^{2}\left(g_{2}\right)\right), \quad g_{1}, g_{2} \in K .
$$

Let $U$ be an arbitrary orthogonal representation of the group $K$ in a real finite-dimensional linear space $\mathrm{V}$ with an inner product $(\cdot, \cdot)$, and let $O$ be a place in $E$. A $\mathrm{V}$-valued field $\mathrm{C}(A)$ 
is called strictly isotropic with respect to $O$ if for any $g \in K$ the random fields $\mathrm{C}(g \cdot A)$ and $U(g) \mathrm{C}(A)$ have the same finite-dimensional distributions. If in addition the random field $\mathrm{C}(A)$ is strictly homogeneous, then it is strictly isotropic with respect to any place.

Assume that the random field $\mathrm{C}(A)$ is second-order, that is

$$
\mathrm{E}\left[\|\mathrm{C}(A)\|^{2}\right]<\infty, \quad A \in E .
$$

Define the one-point correlation tensor of the field $\mathrm{C}(A)$ by

$$
\langle\mathrm{C}(A)\rangle=\mathrm{E}[\mathrm{C}(A)]
$$

and its two-point correlation tensor by

$$
\langle\mathrm{C}(A), \mathrm{C}(B)\rangle=\mathrm{E}[(\mathrm{C}(A)-\langle\mathrm{C}(A)|) \otimes(\mathrm{C}(B)-\langle\mathrm{C}(B)|)] .
$$

Assume that the field $\mathrm{C}(A)$ is mean-square continuous, that is, its two-point correlation tensor $\langle\mathrm{C}(A), \mathrm{C}(B)\rangle: E \times E \rightarrow \mathrm{V} \otimes \mathrm{V}$ is a continuous function. If the field $\mathrm{C}(A)$ is strictly homogeneous, then its one-point correlation tensor is a constant tensor in $\mathrm{V}$, while its twopoint correlation tensor is a function of the vector $B-A$, i.e., a function on $V$. Call such a field wide-sense homogeneous.

Similarly, if the field $\mathrm{C}(A)$ is strictly isotropic, then we have

$$
\begin{aligned}
\langle\mathrm{C}(g \cdot A)\rangle & =U(g)\langle\mathrm{C}(A)\rangle, \\
\langle\mathrm{C}(g \cdot A), \mathrm{C}(g \cdot B)\rangle & =(U \otimes U)(g)\langle\mathrm{C}(A), \mathrm{C}(B)\rangle .
\end{aligned}
$$

Call such a field wide-sense isotropic. In what follows, we consider only wide-sense homogeneous and isotropic random fields and omit the words "wide-sense".

For simplicity, identify the field $\{\mathrm{C}(A): A \in E\}$ defined on $E$ with the field $\left\{\mathrm{C}^{\prime}(\mathbf{x}): \mathbf{x} \in\right.$ $V\}$ defined by $\mathrm{C}^{\prime}(\mathbf{x})=\mathrm{C}(O+\mathbf{x})$. Introduce the Cartesian coordinate system $(x, y, z)$ in $V$. Use the introduced system to identify $V$ with the coordinate space $\mathbb{R}^{3}$ and $\mathrm{O}(V)$ with $\mathrm{O}(3)$. The action of $\mathrm{O}(3)$ on $\mathbb{R}^{3}$ is the matrix-vector multiplication.

Forte and Vianello [7] proved the existence of 8 symmetry classes of elasticity tensors, or elasticity classes. In other words, consider the action

$$
g \cdot \mathrm{C}=\mathrm{S}^{2}\left(\mathrm{~S}^{2}(g)\right) \mathrm{C}
$$

of the group $K=\mathrm{O}(3)$ in the space $\mathrm{V}=\mathrm{S}^{2}\left(\mathrm{~S}^{2}\left(\mathbb{R}^{3}\right)\right)$. The symmetry group of an elasticity tensor $\mathrm{C} \in \mathrm{V}$ is

$$
K(\mathrm{C})=\{g \in \mathrm{O}(V): g \cdot \mathrm{C}=\mathrm{C}\} .
$$

Note that the symmetry group $K(g \cdot \mathrm{C})$ is conjugate through $g$ to $K(\mathrm{C})$ :

$$
K(g \cdot \mathrm{C})=\left\{g h g^{-1}: h \in K(\mathrm{C})\right\} .
$$

Whenever two bodies can be rotated so that their symmetry groups coincide, they share the same symmetry class. Mathematically, two elasticity tensors $\mathrm{C}_{1}$ and $\mathrm{C}_{2}$ are equivalent if and only if there is $g \in \mathrm{O}(3)$ such that $K\left(\mathrm{C}_{1}\right)=K\left(g \cdot \mathrm{C}_{2}\right)$. In view of (5), $\mathrm{C}_{1}$ and $\mathrm{C}_{2}$ are equivalent if and only if their symmetry groups are conjugate. The equivalence classes of the above relation are called the elasticity classes.

The first column of Table 1 adapted from [2], contains the name of an elasticity class. The second column represents a collection of subgroups $H$ of $\mathrm{O}(3)$ such that $H$ is conjugate 
Table 1 Elasticity classes

\begin{tabular}{lll}
\hline Elasticity class & $H$ & $N(H)$ \\
\hline Triclinic & $Z_{2}^{c}$ & $\mathrm{O}(3)$ \\
Monoclinic & $Z_{2} \times Z_{2}^{c}$ & $\mathrm{O}(2) \times Z_{2}^{c}$ \\
Orthotropic & $D_{2} \times Z_{2}^{c}$ & $\mathscr{O} \times Z_{2}^{c}$ \\
Trigonal & $D_{3} \times Z_{2}^{c}$ & $D_{6} \times Z_{2}^{c}$ \\
Tetragonal & $D_{4} \times Z_{2}^{c}$ & $D_{8} \times Z_{2}^{c}$ \\
Transverse isotropic & $\mathrm{O}(2) \times Z_{2}^{c}$ & $\mathrm{O}(2) \times Z_{2}^{c}$ \\
Cubic & $\mathscr{O} \times Z_{2}^{c}$ & $\mathscr{O} \times Z_{2}^{c}$ \\
Isotropic & $\mathrm{O}(3)$ & $\mathrm{O}(3)$ \\
\hline
\end{tabular}

to a symmetry group of any elasticity tensor of the given class. In other words, the above symmetry group lies in the conjugacy class $[H]$ of the group $H$. The third column contains the notation for the normaliser $N(H)$ :

$$
N(H)=\left\{g \in \mathrm{O}(3): g H g^{-1}=H\right\} .
$$

Here $Z_{2}^{c}=\{I,-I\}$, where $I$ is the $3 \times 3$ identity matrix, $Z_{n}$ is generated by the rotation about the $z$-axis with angle $2 \pi / n, \mathrm{O}(2)$ is generated by rotations about the $z$-axis with angle $\theta, 0 \leq \theta<2 \pi$ and the rotation about the $x$-axis with angle $\pi, D_{n}$ is the dihedral group generated by $Z_{n}$ and the rotation about the $x$-axis with angle $\pi$, and $\mathscr{O}$ is the octahedral group which fixes an octahedron. See also [26, Appendix B] for the correspondence between the above notation and notation of Hermann-Mauguin [16, 23] and Schönfließ [32].

The importance of the group $N(H)$ can be clarified as follows. Consider the fixed point set of $H$ :

$$
\mathrm{V}^{H}=\{\mathrm{C} \in \mathrm{V}: g \cdot \mathrm{C}=\mathrm{C} \text { for all } g \in H\} .
$$

By [2, Lemma 3.1], if $H$ is the symmetry group of some tensor $\mathrm{C} \in \mathrm{V}$, then $N(H)$ is the maximal subgroup of $\mathrm{O}(3)$ which leaves $\mathrm{V}^{H}$ invariant. In the language of the representation theory, $\mathrm{V}^{H}$ is an invariant subspace of the representation $g \mapsto \mathrm{S}^{2}\left(\mathrm{~S}^{2}(g)\right)$ of any group $K$ that lies between $H$ and $N(H)$, that is, $\mathrm{S}^{2}\left(\mathrm{~S}^{2}(g)\right) \mathrm{C} \in \mathrm{V}^{H}$ for all $g \in K$ and for all $\mathrm{C} \in \mathrm{V}^{H}$. Denote by $U(g)$ the restriction of the above representation to $\mathrm{V}^{H}$.

The problem is formulated as follows. For each elasticity class $[H]$ and for each group $K$ that lies between $H$ and $N(H)$, consider an $\mathrm{V}^{H}$-valued homogeneous random field $\mathrm{C}(\mathbf{x})$ on $\mathbb{R}^{3}$. Assume that $\mathrm{C}(\mathbf{x})$ is isotropic with respect to $U$ :

$$
\begin{aligned}
\langle\mathrm{C}(g \mathbf{x})\rangle & =U(g)\langle\mathrm{C}(\mathbf{x})\rangle, \\
\langle\mathrm{C}(g \mathbf{x}), \mathrm{C}(g \mathbf{y})\rangle & =(U \otimes U)(g)\langle\mathrm{C}(\mathbf{x}), \mathrm{C}(\mathbf{y})\rangle .
\end{aligned}
$$

We would like to find the general form of the one-and two-point correlation tensors of such a field and the spectral expansion of the field itself in terms of stochastic integrals.

To explain what we mean consider the simplest case when the answer is known. Put $K=H=\mathrm{O}(3), \mathrm{V}^{H}=\mathbb{R}^{1}$, and $U(g)=1$, the trivial representation of $K$. Recall that a measure $\Phi$ on the $\sigma$-field of Borel sets of a Hausdorff topological space $X$ is called tight if for any Borel set $B, \Phi(B)$ is the supremum of $\Phi(K)$ over all compact subsets $K$ of $B$. A measure $\Phi$ is called locally finite if every point of $X$ has a neighbourhood $U$ for which $\Phi(U)$ is finite. A measure $\Phi$ is called a Radon measure if it is tight and locally finite. In what follows we consider only Radon measures and call them just measures. 
Schoenberg [31] proved that the equation

$$
\langle\tau(\mathbf{x}), \tau(\mathbf{y})\rangle=\int_{0}^{\infty} \frac{\sin (\lambda\|\mathbf{y}-\mathbf{x}\|)}{\lambda\|\mathbf{y}-\mathbf{x}\|} \mathrm{d} \Phi(\lambda)
$$

establishes a one-to-one correspondence between the class of two-point correlation tensors of homogeneous and isotropic random fields $\tau(\mathbf{x})$ and the class of finite measures on $[0, \infty)$.

Let $L_{0}^{2}(\Omega)$ be the Hilbert space of centred complex-valued random variables with finite variance. Let $Z$ be a $L_{0}^{2}(\Omega)$-valued measure on the $\sigma$-field of Borel sets of a Hausdorff topological space $X$. A measure $\Phi$ is called the control measure for $Z$, if for any Borel sets $B_{1}$ and $B_{2}$ we have

$$
\mathrm{E}\left[\overline{Z\left(B_{1}\right)} Z\left(B_{2}\right)\right]=\Phi\left(B_{1} \cap B_{2}\right) .
$$

Yaglom [37] and independently M.I. Yadrenko in his unpublished $\mathrm{PhD}$ thesis proved that the field $\tau(\mathbf{x})$ has the form

$$
\tau(\rho, \theta, \varphi)=C+\pi \sqrt{2} \sum_{\ell=0}^{\infty} \sum_{m=-\ell}^{\ell} S_{\ell}^{m}(\theta, \varphi) \int_{0}^{\infty} \frac{J_{\ell+1 / 2}(\lambda \rho)}{\sqrt{\lambda \rho}} \mathrm{d} Z_{\ell}^{m}(\lambda),
$$

where $C=\langle\tau(\mathbf{x})\rangle \in \mathbb{R}^{1},(\rho, \theta, \varphi)$ are spherical coordinates in $\mathbb{R}^{3}, S_{\ell}^{m}(\theta, \varphi)$ are real-valued spherical harmonics, $J_{\ell+1 / 2}(\lambda \rho)$ are the Bessel functions of the first kind of order $\ell+1 / 2$, and $Z_{\ell}^{m}$ is a sequence of centred uncorrelated real-valued orthogonal random measures on $[0, \infty)$ with the measure $\Phi$ as their common control measure.

Other known results include the case of $\mathrm{V}^{H}=\mathbb{R}^{3}$, and $U(g)=g$. Yaglom [36] found the general form of the two-point correlation tensor. Malyarenko and Ostoja-Starzewski [22] found the spectral expansion of the field. In the same paper, they found both the general form of the two-point correlation tensor and the spectral expansion of the field for the case of $\mathrm{V}^{H}=\mathrm{S}^{2}\left(\mathbb{R}^{3}\right)$, and $U(g)=\mathrm{S}^{2}(g)$. In [20] they solved one of the cases for two-dimensional elasticity, when $V=\mathbb{R}^{2}, K=\mathrm{O}(2), \mathrm{V}^{H}=\mathrm{S}^{2}\left(\mathrm{~S}^{2}\left(\mathbb{R}^{2}\right)\right)$, and $U(g)=\mathrm{S}^{2}\left(\mathrm{~S}^{2}(g)\right)$.

Remark 1 Another approach to studying tensor-valued random fields was elaborated by Guilleminot and Soize [11-15]. Using a stochastic model alternative to our model, they constructed a generator for random fields that have prescribed symmetry properties, take values in the set of symmetric nonnegative-definite tensors, depend on a few real parameters, and may be easily simulated and calibrated. The question of constructing a generator with similar properties based on the stochastic model described below raises several interesting issues and will be considered in forthcoming publications.

\section{A General Result}

The idea of this Section is as follows. Let $\mathrm{V}$ be a finite-dimensional real linear space, let $K$ be a closed subgroup of the group $\mathrm{O}(3)$, and let $U$ be an orthogonal representation of the group $K$ in the space $\mathrm{V}$. Consider a homogeneous and isotropic random field $\mathrm{C}(\mathbf{x}), \mathbf{x} \in \mathbb{R}^{3}$, and solve the problem formulated in Section 2. In Section 5, apply general formulae to our cases. The resulting Theorems 1-16 are particular cases of general Theorem 0 .

To obtain general formulae, we describe all homogeneous random fields taking values in $\mathrm{V}$ and throw away non-isotropic ones. The first obstacle here is as follows. The complete description of such fields is unknown. We use the following result instead. 
Let $\mathrm{V}^{\mathbb{C}}$ be a complex finite-dimensional linear space with an inner product $(\cdot, \cdot)$ that is linear in the second argument, as is usual in physics. Let $J$ be a real structure on $\mathbb{V}^{\mathbb{C}}$, that is, a map $J: \mathbb{V}^{\mathbb{C}} \rightarrow \mathbb{V}^{\mathbb{C}}$ satisfying the following conditions:

$$
\begin{aligned}
J\left(\alpha \mathrm{C}_{1}+\beta \mathrm{C}_{2}\right) & =\bar{\alpha} J\left(\mathrm{C}_{1}\right)+\bar{\beta} J\left(\mathrm{C}_{2}\right), \\
J(J(\mathrm{C})) & =\mathrm{C}
\end{aligned}
$$

for all $\alpha, \beta \in \mathbb{C}$ and for all $\mathrm{C}_{1}, \mathrm{C}_{2} \in \mathrm{V}^{\mathbb{C}}$. In other words, $J$ is a multidimensional and coordinate-free generalisation of complex conjugation. The set of all eigenvectors of $J$ that correspond to eigenvalue 1 , constitute a real linear space, denote it by $\mathrm{V}$. Let $\mathrm{H}$ be the real linear space of Hermitian linear operators in $\mathrm{V}^{\mathbb{C}}$. The real structure $J$ induces a linear operator $\mathrm{J}$ in $\mathrm{H}$. For any $A \in \mathrm{H}$, the operator $\mathrm{J} A$ acts by

$$
(\mathrm{J} A) \mathrm{C}=J(A J \mathrm{C}), \quad \mathrm{C} \in \mathrm{V}^{\mathbb{C}} .
$$

In coordinates, the operator $\mathrm{J}$ is just the transposition of a matrix.

The result by Cramér [5] in coordinate-free form is formulated as follows. Equation

$$
\langle\mathrm{C}(\mathbf{x}), \mathrm{C}(\mathbf{y})\rangle=\int_{\hat{\mathbb{R}}^{3}} \mathrm{e}^{\mathrm{i}(\mathbf{p}, \mathbf{y}-\mathbf{x})} \mathrm{d} F(\mathbf{p})
$$

establishes a one-to-one correspondence between the class of two-point correlation tensors of homogeneous mean-square continuous $\mathbb{V}^{\mathbb{C}}$-valued random fields $\mathbf{C}(\mathbf{x})$ and the class of measures on the $\sigma$-field of Borel sets of the wavenumber domain $\hat{\mathbb{R}}^{3}$ tasking values in the set of nonnegative-definite Hermitian linear operators in $\mathbb{V}^{\mathbb{C}}$. For $\mathrm{V}$-valued random fields, there is only a necessary condition: if $\mathrm{C}(\mathbf{x})$ is $\mathrm{V}$-valued, then the measure $F$ satisfies

$$
F(-B)=\mathrm{J} F(B), \quad B \in \mathfrak{B}\left(\hat{\mathbb{R}}^{3}\right),
$$

where $-B=\{-\mathbf{p}: \mathbf{p} \in B\}$.

Introduce the trace measure $\mu$ by $\mu(B)=\operatorname{tr} F(B), B \in \mathfrak{B}\left(\hat{\mathbb{R}}^{3}\right)$ and note that $F$ is absolutely continuous with respect to $\mu$. This means that (7) may be written as

$$
\langle\mathrm{C}(\mathbf{x}), \mathrm{C}(\mathbf{y})\rangle=\int_{\hat{\mathbb{R}}^{3}} \mathrm{e}^{\mathrm{i}(\mathbf{p}, \mathbf{y}-\mathbf{x})} f(\mathbf{p}) \mathrm{d} \mu(\mathbf{p}),
$$

where $f(\mathbf{p})$ is a measurable function on the wavenumber domain taking values in the set of all nonnegative-definite Hermitian linear operators in $\mathbb{V}^{\mathbb{C}}$ with unit trace, that satisfies the following condition

$$
f(-\mathbf{p})=J f(\mathbf{p}) .
$$

Using representation theory, it is possible to prove the following. Let $\mathrm{C}_{1}, \mathrm{C}_{2} \in \mathrm{V}$. Let $L\left(\mathrm{C}_{1} \otimes \mathrm{C}_{2}\right)$ be the operator in $\mathrm{H}$ acting on a tensor $\mathrm{C} \in \mathrm{V}^{\mathbb{C}}$ by

$$
L\left(\mathrm{C}_{1} \otimes \mathrm{C}_{2}\right) \mathrm{C}=\left(J \mathrm{C}_{1}, \mathrm{C}\right) \mathrm{C}_{2} .
$$

By linearity, this action may be extended to an isomorphism $L$ between $\mathrm{V} \otimes \mathrm{V}$ and $\mathrm{H}$. The orthogonal operators $L U \otimes U(g) L^{-1}, g \in K$, constitute an orthogonal representation of the group $K$ in the space $\mathrm{H}$, equivalent to the tensor square $U \otimes U$ of the representation $U$. The operator $L$ is an intertwining operator between the spaces $\mathrm{V} \otimes \mathrm{V}$ and $\mathrm{H}$ where equivalent 
representations $U \otimes U$ and $L U \otimes U L^{-1}$ act. In what follows, we are working only with the latter representation, for simplicity denote it again by $U \otimes U$ and note that it acts in the space $\mathrm{H}$ by

$$
(U \otimes U)(g) A=U(g) A U^{-1}(g), \quad A \in \mathrm{H} .
$$

Denote $\mathrm{H}_{+}=L S^{2}(V)$. In coordinates, it is the subspace of Hermitian matrices with realvalued matrix entries. If $-I \in K$, then the second equation in (6) and (8) together are equivalent to the following conditions:

$$
\mu(g B)=\mu(B), \quad B \in \mathfrak{B}\left(\hat{\mathbb{R}}^{3}\right)
$$

and

$$
f(\mathbf{p}) \in \mathrm{H}_{+}, \quad f(g \mathbf{p})=\mathrm{S}^{2}(U(g)) f(\mathbf{p}) .
$$

The description of all measures $\mu$ satisfying (9) is well known, see [3]. There are finitely many, say $M$, orbit types for the action of $K$ in $\hat{\mathbb{R}}^{3}$ by

$$
(g \mathbf{p}, \mathbf{x})=\left(\mathbf{p}, g^{-1} \mathbf{x}\right) .
$$

Denote by $\left(\hat{\mathbb{R}}^{3} / K\right)_{m}, 0 \leq m \leq M-1$ the set of all orbits of the $m$ th type. It is known, see [2], that all the above sets are manifolds. Assume for simplicity of notation that there are charts $\lambda_{m}$ such that the domain of $\lambda_{m}$ is dense in $\left(\hat{\mathbb{R}}^{3} / K\right)_{m}$. The orbit of the $m$ th type is the manifold $K / H_{m}$, where $H_{m}$ is a stationary subgroup of a point on the orbit. Assume that the domain of a chart $\varphi_{m}$ is a dense set in $K / H_{m}$, and let $\mathrm{d} \boldsymbol{\varphi}_{m}$ be the unique probabilistic $K$-invariant measure on the $\sigma$-field of Borel sets of $K / H_{m}$. There are the unique measures $\Phi_{m}$ on the $\sigma$-fields of Borel sets in $\left(\hat{\mathbb{R}}^{3} / K\right)_{m}$ such that

$$
\int_{\hat{\mathbb{R}}^{3}} \mathrm{e}^{\mathrm{i}(\mathbf{p}, \mathbf{y}-\mathbf{x})} f(\mathbf{p}) \mathrm{d} \mu(\mathbf{p})=\sum_{m=0}^{M-1} \int_{\left(\hat{\mathbb{R}}^{3} / K\right)_{m}} \int_{K / H_{m}} \mathrm{e}^{\mathrm{i}\left(\left(\lambda_{m}, \boldsymbol{\varphi}_{m}\right), \mathbf{y}-\mathbf{x}\right)} f\left(\boldsymbol{\lambda}_{m}, \boldsymbol{\varphi}_{m}\right) \mathrm{d} \boldsymbol{\varphi}_{m} \mathrm{~d} \Phi_{m}\left(\boldsymbol{\lambda}_{m}\right) .
$$

To find all functions $f$ satisfying (10), proceed as follows. Fix an orbit $\lambda_{m}$ and denote by $\varphi_{m}^{0}$ the coordinates of the intersection of the orbit $\lambda_{m}$ with the set $\left(\hat{\mathbb{R}}^{3} / K\right)_{m}$. Let $U^{m}$ be the restriction of the representation $S^{2}(U)$ to the group $H_{m}$. We have $g\left(\lambda_{m}, \varphi_{m}^{0}\right)=\left(\lambda_{m}, \varphi_{m}^{0}\right)$ for all $g \in H_{m}$, because $H_{m}$ is the stationary subgroup of the point $\left(\lambda_{m}, \varphi_{m}^{0}\right)$. For $g \in H_{m}$, (10) becomes

$$
f\left(\lambda_{m}, \varphi_{m}^{0}\right)=U^{m}(g) f\left(\lambda_{m}, \varphi_{m}^{0}\right) .
$$

Any orthogonal representation of a compact topological group in a space $\mathrm{H}$ has at least two invariant subspaces: $\{0\}$ and $\mathrm{H}$. The representation is called irreducible if no other invariant subspaces exist. The space of any finite-dimensional orthogonal representation of a compact topological group can be uniquely decomposed into a direct sum of isotypic subspaces. Each isotypic subspace is the direct sum of finitely many subspaces where the copies of the same irreducible representation act. Equation (11) means that the operator $f\left(\lambda_{m}, \varphi_{m}^{0}\right)$ lies in the isotypic subspace $\mathrm{H}_{m}$ which corresponds to the trivial representation of the group $H_{m}$. The intersection of this subspace with the convex compact set of all nonnegative-definite operators in $\mathrm{H}_{+}$with unit trace is again a convex compact set, call it $\mathscr{C}_{m}$. As $\lambda_{m}$ runs over $\left(\mathbb{R}^{3} / K\right)_{m}, f\left(\lambda_{m}, \varphi_{m}^{0}\right)$ becomes an arbitrary measurable function taking values in $\mathscr{C}_{m}$.

An irreducible orthogonal representation of the group $K$ is called a representation of class 1 with respect to the group $H_{m}$ if the restriction of this representation to $H_{m}$ contains at least one copy of the trivial representation of $H_{m}$. Let $\mathrm{S}^{2}(U)_{m}$ be the restriction 
of the representation $S^{2}(U)$ to the direct sum of the isotypic subspaces of the irreducible representation of class 1 with respect to $H_{m}$. Let $g_{\varphi_{m}}$ be an arbitrary element of $K$ such that $g_{\varphi_{m}}\left(\varphi_{m}^{0}\right)=\varphi_{m}$. Two such elements differ by an element of $H_{m}$, therefore the second equation in (10) becomes

$$
f\left(\lambda_{m}, \boldsymbol{\varphi}_{m}\right)=\mathrm{S}^{2}\left(U\left(g_{\varphi_{m}}\right)\right)_{m} f\left(\lambda_{m}, \boldsymbol{\varphi}_{m}^{0}\right)
$$

The two-point correlation tensor of the field takes the form

$$
\begin{aligned}
\langle\mathrm{C}(\mathbf{x}), \mathrm{C}(\mathbf{y})\rangle= & \sum_{m=0}^{M-1} \int_{\left(\hat{\mathbb{R}}^{3} / K\right)_{m}} \int_{K / H_{m}} \mathrm{e}^{\mathrm{i}\left(g_{\varphi_{m}}\left(\lambda_{m}, \varphi_{m}^{0}\right), \mathbf{y}-\mathbf{x}\right)} \mathrm{S}^{2}\left(U\left(g_{\boldsymbol{\varphi}_{m}}\right)\right)_{m} \\
& \times f\left(\lambda_{m}, \boldsymbol{\varphi}_{m}^{0}\right) \mathrm{d} \boldsymbol{\varphi}_{m} \mathrm{~d} \Phi_{m}\left(\lambda_{m}\right) .
\end{aligned}
$$

Choose an orthonormal basis $\mathrm{T}^{1}, \ldots, \mathrm{T}^{\mathrm{dim} \mathrm{V}}$ in the space $\mathrm{V}$. The tensor square $\mathrm{V} \otimes \mathrm{V}$ has several orthonormal bases. The coupled basis consists of tensor products $\mathrm{T}^{i} \otimes \mathrm{T}^{j}, 1 \leq i$, $j \leq \operatorname{dim} \mathrm{V}$. The $m$ th uncoupled basis is build as follows. Let $U^{m, 1}, \ldots, U^{m, k_{m}}$ be all nonequivalent irreducible orthogonal representations of the group $K$ of class 1 with respect to $H_{m}$ such that the representation $\mathrm{S}^{2}(U)$ contains isotypic subspaces where $c_{m k}$ copies of the representation $U^{m, k}$ act, and let the restriction of the representation $U^{m, k}$ to $H_{m}$ contains $d_{m k}$ copies of the trivial representation of $H_{m}$. Let $\mathrm{T}^{m k l n}, 1 \leq l \leq d_{m k}, 1 \leq n \leq c_{m k}$ be an orthonormal basis in the space where the $n$th copy act. Complete the above basis to the basis $T^{m k l n}, 1 \leq l \leq \operatorname{dim} U^{m, k}$ and call this basis the $m$ th uncoupled basis. The vectors of the coupled basis are linear combinations of the vectors of the $m$ th uncoupled basis:

$$
\mathrm{T}^{i} \otimes \mathrm{T}^{j}=\sum_{k=1}^{k_{m}} \sum_{l=1}^{\operatorname{dim} U^{m, k}} \sum_{n=1}^{c_{m k}} c_{i j}^{m k l n} \mathrm{~T}^{m k l n}+\cdots
$$

where dots denote the terms that include the tensors in the basis of the space $S^{2}(V) \ominus S^{2}(V)_{m}$. In the introduced coordinates, (12) takes the form

$$
\begin{aligned}
\langle\mathbf{C}(\mathbf{x}), \mathbf{C}(\mathbf{y})\rangle_{i j}= & \sum_{m=0}^{M-1} \sum_{k=1}^{k_{m}} \sum_{l=1}^{\operatorname{dim} U^{m, k}} \sum_{l^{\prime}=1}^{d_{m k}} \sum_{n=1}^{c_{m k}} c_{i j}^{m k l n} \int_{\left(\hat{\mathbb{R}}^{3} / K\right)_{m}} \int_{K / H_{m}} \mathrm{e}^{\mathrm{i}\left(g_{\boldsymbol{\varphi}_{m}}\left(\boldsymbol{\lambda}_{m}, \boldsymbol{\varphi}_{m}^{0}\right), \mathbf{y}-\mathbf{x}\right)} \\
& \times U_{l l^{\prime}}^{m, k}\left(\boldsymbol{\varphi}_{m}\right) f_{l^{\prime} n}\left(\boldsymbol{\lambda}_{m}, \boldsymbol{\varphi}_{m}^{0}\right) \mathrm{d} \boldsymbol{\varphi}_{m} \mathrm{~d} \Phi_{m}\left(\boldsymbol{\lambda}_{m}\right) .
\end{aligned}
$$

The choice of bases inside the isotypic subspaces is not unique. One has to choose them in such a way that calculation of the transition coefficients $c_{i j}^{m k l n}$ is as easy as possible.

To calculate the inner integrals, we proceed as follows. Consider the action of $K$ on $\mathbb{R}^{3}$ by matrix-vector multiplication. Let $\left(\mathbb{R}^{3} / K\right)_{m}, 0 \leq m \leq M-1$ be the set of all orbits of the $m$ th type. Let $\boldsymbol{\rho}_{m}$ be such a chart that its domain is dense in $\left(\mathbb{R}^{3} / K\right)_{m}$. Let $\boldsymbol{\psi}_{m}$ be a chart in $K / H_{m}$ with a dense domain, and let $\mathrm{d} \boldsymbol{\psi}_{m}$ be the unique probabilistic $K$-invariant measure on the $\sigma$-field of Borel sets of $K / H_{m}$. It is known that the sets of orbits of one of the types, say $\left(\hat{\mathbb{R}}^{3} / K\right)_{M-1}$ (resp. $\left.\left(\mathbb{R}^{3} / K\right)_{M-1}\right)$, are dense in $\hat{\mathbb{R}}^{3}$ (resp. $\mathbb{R}^{3}$ ). Write the plane wave $\mathrm{e}^{\mathrm{i}\left(g_{\varphi_{M-1}}\left(\lambda_{M-1}, \varphi_{M-1}^{0}\right), \mathbf{y}-\mathbf{x}\right)}$ as

$$
\mathrm{e}^{\mathrm{i}\left(g_{\boldsymbol{\varphi}_{M-1}}\left(\lambda_{M-1}, \varphi_{M-1}^{0}\right), \mathbf{y}-\mathbf{x}\right)}=\mathrm{e}^{\mathrm{i}\left(g_{\varphi_{M-1}}\left(\lambda_{M-1}, \varphi_{M-1}^{0}\right), g_{\psi_{M-1}}\left(\rho_{M-1}, \psi_{M-1}^{0}\right)\right)},
$$


and consider the plane wave as a function of two variables $\varphi_{M-1}$ and $\psi_{M-1}$ with domain $\left(K / H_{M-1}\right)^{2}$. This function is $K$-invariant:

$$
\mathrm{e}^{\mathrm{i}\left(g g_{\varphi_{M-1}}\left(\lambda_{M-1}, \varphi_{M-1}^{0}\right), g g_{\psi_{M-1}}\left(\rho_{M-1}, \psi_{M-1}^{0}\right)\right)}=\mathrm{e}^{\mathrm{i}\left(g_{\varphi_{M-1}}\left(\lambda_{M-1}, \varphi_{M-1}^{0}\right), g_{\psi_{M-1}}\left(\rho_{M-1}, \psi_{M-1}^{0}\right)\right)}, \quad g \in K .
$$

Denote by $\hat{K}_{H_{M-1}}$ the set of all equivalence classes of irreducible representations of $K$ of class 1 with respect to $H_{M-1}$, and let the restriction of the representation $U^{q} \in \hat{K}_{H_{M-1}}$ to $H_{M-1}$ contains $d_{q}$ copies of the trivial representation of $H_{M-1}$. By the Fine Structure Theorem [17], there are some numbers $d_{q}^{\prime} \leq d_{q}$ such that the set

$$
\left\{\operatorname{dim} U^{q} \cdot U_{l l^{\prime}}^{q}\left(\boldsymbol{\varphi}_{M-1}\right) U_{l l^{\prime}}^{q}\left(\boldsymbol{\psi}_{M-1}\right): U^{q} \in \hat{K}_{H_{M-1}}, 1 \leq l \leq \operatorname{dim} U^{q}, 1 \leq l^{\prime} \leq d_{q}^{\prime}\right\}
$$

is the orthonormal basis in the Hilbert space $L^{2}\left(\left(K / H_{M-1}\right)^{2}, \mathrm{~d} \boldsymbol{\varphi}_{M-1} \mathrm{~d} \boldsymbol{\psi}_{M-1}\right)$. Let

$$
\begin{aligned}
j_{l l^{\prime}}^{q}\left(\boldsymbol{\lambda}_{M-1}, \boldsymbol{\rho}_{M-1}\right)= & \operatorname{dim} U^{q} \int_{\left(K / H_{M-1}\right)^{2}} \mathrm{e}^{\mathrm{i}\left(g_{\varphi_{M-1}}\left(\lambda_{M-1}, \boldsymbol{\varphi}_{M-1}^{0}\right), g_{\psi_{M-1}}\left(\rho_{M-1}, \boldsymbol{\psi}_{M-1}^{0}\right)\right)} \\
& \times U_{l l^{\prime}}^{q}\left(\boldsymbol{\varphi}_{M-1}\right) U_{l l^{\prime}}^{q}\left(\boldsymbol{\psi}_{M-1}\right) \mathrm{d} \boldsymbol{\varphi}_{M-1} \mathrm{~d} \boldsymbol{\psi}_{M-1}
\end{aligned}
$$

be the corresponding Fourier coefficients. The uniformly convergent Fourier expansion takes the form

$$
\begin{aligned}
\mathrm{e}^{\mathrm{i}\left(g_{\varphi_{M-1}}\left(\lambda_{M-1}, \boldsymbol{\varphi}_{M-1}^{0}\right), g_{\boldsymbol{\psi}_{M-1}}\left(\boldsymbol{\rho}_{M-1}, \boldsymbol{\psi}_{M-1}^{0}\right)\right)}= & \sum_{U^{q} \in \hat{K}_{H_{M-1}}} \sum_{l=1}^{\operatorname{dim} U^{q}} \sum_{l^{\prime}=1}^{d_{q}^{\prime}} \operatorname{dim} U^{q} \\
& \times j_{l l^{\prime}}^{q}\left(\boldsymbol{\lambda}_{M-1}, \boldsymbol{\rho}_{M-1}\right) U_{l l^{\prime}}^{q}\left(\boldsymbol{\varphi}_{M-1}\right) U_{l l^{\prime}}^{q}\left(\boldsymbol{\psi}_{M-1}\right) .
\end{aligned}
$$

This expansion is defined on the dense set

$$
\left(\hat{\mathbb{R}}^{3} / K\right)_{M-1} \times\left(K / H_{M-1}\right) \times\left(\mathbb{R}^{3} / K\right)_{M-1} \times\left(K / H_{M-1}\right)
$$

and may be extended to all of $\hat{\mathbb{R}}^{3} \times \mathbb{R}^{3}$ by continuity. Substituting the extended expansion to (13), we obtain the expansion

$$
\begin{aligned}
\langle\mathrm{C}(\mathbf{x}), \mathrm{C}(\mathbf{y})\rangle_{i j}= & \sum_{m=0}^{M-1} \sum_{k=1}^{k_{m}} \sum_{l=1}^{\operatorname{dim} U^{m, k}} \sum_{l^{\prime}=1}^{d_{m k}^{\prime}} \sum_{n=1}^{c_{m k}} c_{i j}^{m k l n} \int_{\left(\hat{\mathbb{R}}^{3} / K\right)_{m}} j_{l l^{\prime}}^{q}\left(\boldsymbol{\lambda}_{m}, \boldsymbol{\rho}_{0}\right) \\
& \times U_{l l^{\prime}}^{m, k}\left(\boldsymbol{\psi}_{m}\right) f_{l^{\prime} n}\left(\boldsymbol{\lambda}_{m}, \boldsymbol{\varphi}_{m}^{0}\right) \mathrm{d} \Phi_{m}\left(\boldsymbol{\lambda}_{m}\right) .
\end{aligned}
$$

Theorem 0 Let $-I \in K$. The one-point correlation tensor of a homogeneous and $(K, U)$ isotropic random field lies in the space of the isotypic component of the representation $U$ that corresponds to the trivial representation of $K$ and is equal to 0 if no such isotypic component exists. Its two-point correlation tensor is given by (15).

Remark 2 The results by [20, 22, 31, 36, 37] as well as Theorems 1-16 below are particular cases of Theorem 0 . The expansion (15) is the first necessary step in studying random fields connected to Hooke's law. 
Later we will see that it is easy to write the spectral expansion of the field directly if the group $K$ is finite. Otherwise, we write the Fourier expansion (14) for plane waves $\mathrm{e}^{\mathrm{i}(\mathbf{p}, \mathbf{y})}$ and $\mathrm{e}^{-\mathrm{i}(\mathbf{p}, \mathbf{x})}$ separately and substitute both expansions to (13). As a result, we obtain the expansion of the two-point correlation tensor of the field in the form

$$
\langle\mathrm{C}(\mathbf{x}), \mathrm{C}(\mathbf{y})\rangle_{i j}=\int_{\Lambda} \overline{u(\mathbf{x}, \lambda)} u(\mathbf{y}, \lambda) \mathrm{d} \Phi_{i j}(\lambda),
$$

where $\Lambda$ is a set, and where $F$ is a measure on a $\sigma$-field $\mathfrak{L}$ of subsets of $\Lambda$ taking values in the set of Hermitian nonnegative-definite operators on $\mathbb{V}^{\mathbb{C}}$. Moreover, the set $\left\{u(\mathbf{x}, \lambda): \mathbf{x} \in \mathbb{R}^{3}\right\}$ is total in the Hilbert space $L^{2}(\Lambda, \Phi)$ of the measurable complex-valued functions on $\Lambda$ that are square-integrable with respect to the measure $\Phi$, that is, the set of finite linear combinations $\sum c_{n} u\left(\mathbf{x}_{n}, \lambda\right)$ is dense in the above space. By Karhunen's theorem [18], the field $\mathbf{C}(\mathbf{x})$ has the following spectral expansion:

$$
\mathrm{C}(\mathbf{x})=\mathrm{E}[\mathrm{C}(\mathbf{0})]+\int_{\Lambda} u(\mathbf{x}, \lambda) \mathrm{dZ}(\lambda),
$$

where $Z$ is a measure on the measurable space $(\Lambda, \mathfrak{L})$ taking values in the Hilbert space of random tensors $\mathrm{Z}: \Omega \rightarrow \mathrm{V}^{\mathbb{C}}$ with $\mathrm{E}[\mathrm{Z}]=0$ and $\mathrm{E}\left[\|\mathrm{Z}\|^{2}\right]<\infty$. The measure $F$ is the control measure of the measure $\mathrm{Z}$, i.e.,

$$
\mathrm{E}\left[J Z(A) Z^{\top}(B)\right]=\Phi(A \cap B), \quad A, B \in \mathfrak{L} .
$$

The components of the random tensor $Z(A)$ are correlated, which creates difficulties when one tries to use (16) for computer simulation. It is possible to use Cholesky decomposition and to write the expansion of the field using uncorrelated random measures, see details in [22].

\section{Preliminary Calculations}

The possibilities for the group $K$ are as follows. In the triclinic class, there exist infinitely many groups between $Z_{2}^{c}$ and $\mathrm{O}(3)$, we put $K_{1}=Z_{2}^{c}$ and $K_{2}=\mathrm{O}(3)$. Similarly, for the monoclinic class put $K_{3}=Z_{2} \times Z_{2}^{c}$ and $K_{4}=\mathrm{O}(2) \times Z_{2}^{c}$. The possibilities for the orthotropic class are $K_{5}=D_{2} \times Z_{2}^{c}, K_{6}=D_{4} \times Z_{2}^{c}, K_{7}=D_{6} \times Z_{2}^{c}, K_{8}=\mathscr{T} \times Z_{2}^{c}$, and $K_{9}=\mathscr{O} \times Z_{2}^{c}$. Here $\mathscr{T}$ is the tetrahedral group which fixes a tetrahedron. In the trigonal class, we have $K_{10}=D_{3} \times Z_{2}^{c}$ and $K_{11}=D_{6} \times Z_{2}^{c}$. In the tetragonal class, the possibilities are $K_{12}=$ $D_{4} \times Z_{2}^{c}$ and $K_{13}=D_{8} \times Z_{2}^{c}$. In the three remaining classes, the possibilities are $K_{14}=$ $\mathrm{O}(2) \times Z_{2}^{c}, K_{15}=\mathscr{O} \times Z_{2}^{c}$, and $K_{16}=\mathrm{O}(3)$. The intermediate groups were determined using [4, Vol. 1, Fig. 10.1.3.2]. For each group $K_{i}, 1 \leq i \leq 16$, we formulate Theorem number $i$ below.

\subsection{The Structure of the Representation $U$}

The notation for irreducible orthogonal representation is as follows. If $K_{i}$ is a finite group, we use the Mulliken notation [24], see also [1, Chapter 14] to denote the irreducible unitary representation of $K_{i}$. For an irreducible orthogonal representation, consider its complexification. A standard result of representation theory, see, for example, [6, Proposition 4.8.4], states that there are three possibilities: 
Table 2 The structure of the representation $U$

\begin{tabular}{lll}
\hline$K_{i}$ & Table number & The structure of $U$ \\
\hline$K_{1}=Z_{2}^{c}$ & 11 & $21 A_{g}$ \\
$K_{2}=\mathrm{O}(3)$ & - & $2 U^{0 g} \oplus 2 U^{2 g} \oplus U^{4 g}$ \\
$K_{3}=Z_{2} \times Z_{2}^{c}$ & 60 & $13 A_{g}$ \\
$K_{4}=\mathrm{O}(2) \times Z_{2}^{c}$ & - & $5 U^{0 g g} \oplus 3 U^{2 g} \oplus U^{4 g}$ \\
$K_{5}=D_{2} \times Z_{2}^{c}$ & 31 & $9 A_{g}$ \\
$K_{6}=D_{4} \times Z_{2}^{c}$ & 33 & $6 A_{1 g} \oplus 3 B_{1 g}$ \\
$K_{7}=D_{6} \times Z_{2}^{c}$ & 35 & $5 A_{1 g} \oplus 2 E_{2 g}$ \\
$K_{8}=\mathscr{T} \times Z_{2}^{c}$ & 72 & $3 A_{g} \oplus 3\left({ }^{1} E_{g} \oplus{ }^{2} E_{g}\right)$ \\
$K_{9}=\mathscr{O} \times Z_{2}^{c}$ & 71 & $3 A_{1 g} \oplus 3 E_{g}$ \\
$K_{10}=D_{3} \times Z_{2}^{c}$ & 42 & $6 A_{1 g}$ \\
$K_{11}=D_{6} \times Z_{2}^{c}$ & 35 & $5 A_{1 g} \oplus B_{1 g}$ \\
$K_{12}=D_{4} \times Z_{2}^{c}$ & 33 & $6 A_{1 g}$ \\
$K_{13}=D_{8} \times Z_{2}^{c}$ & 37 & $5 A_{1 g} \oplus B_{2 g}$ \\
$K_{14}=\mathrm{O}(2) \times Z_{2}^{c}$ & - & $5 U^{0 g g}$ \\
$K_{15}=\mathscr{O} \times Z_{2}^{c}$ & 71 & $3 A_{1 g}$ \\
$K_{16}=\mathrm{O}(3)$ & - & $2 U^{0 g}$
\end{tabular}

- The complexification is irreducible, say $U$. Then, it is a sum of two equivalent orthogonal representations, and we denote each of them by $U$.

- The complexification is a direct sum of two mutually conjugate representation $U_{1}$ and $U_{2}$, that is, $U_{2}(g)=\overline{U_{1}(g)}$. We denote the orthogonal representation by $U_{1} \oplus U_{2}$.

- The complexification is a direct sum of two copies of an irreducible representation $U$. We denote the orthogonal representation by $U \oplus U$.

For infinite groups, the notation is as follows. For $K_{2}=\mathrm{O}(3)$, we denote the representations by $U^{\ell g}$ (the tensor product of the representation $U^{\ell}$ of the group $\mathrm{SO}(3)$ and the trivial representation $A_{g}$ of $Z_{2}^{c}$ ) and $U^{\ell u}$ (that of $U^{\ell}$ and the nontrivial representation $A_{u}$ of $Z_{2}^{c}$ ). For $K_{4}=K_{14}=\mathrm{O}(2) \times Z_{2}^{c}$ the notation is $U^{0 g g}=U^{0 g} \otimes A_{g}, U^{0 g u}=U^{0 g} \otimes A_{u}$, $U^{0 u g}=U^{0 u} \otimes A_{g}, U^{0 u u}=U^{0 u} \otimes A_{u}, U^{\ell g}=U^{\ell} \otimes A_{g}$, and $U^{\ell u}=U^{\ell} \otimes A_{u}$, where $U^{0 g}$ is the trivial representation of $\mathrm{O}(2), U^{0 u}(g)=\operatorname{det} g$, and

$$
\begin{gathered}
U^{\ell}\left(\left(\begin{array}{cc}
\cos (\varphi) & \sin (\varphi) \\
-\sin (\varphi) & \cos (\varphi)
\end{array}\right)\right)=\left(\begin{array}{cc}
\cos (\ell \varphi) & \sin (\ell \varphi) \\
-\sin (\ell \varphi) & \cos (\ell \varphi)
\end{array}\right), \\
U^{\ell}\left(\left(\begin{array}{cc}
\cos (\varphi) & \sin (\varphi) \\
\sin (\varphi) & -\cos (\varphi)
\end{array}\right)\right)=\left(\begin{array}{cc}
\cos (\ell \varphi) & \sin (\ell \varphi) \\
\sin (\ell \varphi) & -\cos (\ell \varphi)
\end{array}\right) .
\end{gathered}
$$

Fist, we determine the structure of the representation $g \mapsto g$ of the group $K_{i}$. For finite groups, the above structure is given in Table $n .10$ in [1], where $n$ in the number given in the second column of Table 2. For $K_{2}$ and $K_{16}$, this representation is $U^{1 u}$, for $K_{4}$ and $K_{14}$ it is $U^{1 u} \oplus U^{0 u u}$. Then we determine the structure of the representations $S^{2}(g)$ and $S^{2}\left(S^{2}(g)\right)$. For finite groups, we use Table $n .8$. For infinite groups, we use the following multiplication rules. The product of two isomorphic irreducible representations of $Z_{2}^{c}$ is $A_{g}$, that of two different representations is $A_{u}$. For $\mathrm{SO}(3)$, we have

$$
U^{\ell_{1}} \otimes U^{\ell_{2}}=\sum_{\ell=\left|\ell_{1}-\ell_{2}\right|}^{\ell_{1}+\ell_{2}} \oplus U^{\ell} .
$$


Table 3 The isotropy subgroups of the groups $K_{i}$

\begin{tabular}{lllllll}
\hline$K_{i}$ & $H_{1}$ & $H_{2}$ & $H_{3}$ & $H_{4}$ & $H_{5}$ & $H_{6}$ \\
\hline$K_{1}$ & $I$ & & & & \\
$K_{2}, K_{16}$ & $\mathrm{O}(2)$ & & & & \\
$K_{3}$ & $Z_{2}$ & $Z_{2}^{-}$ & $I$ & & \\
$K_{4}, K_{14}$ & $\mathrm{O}(2)$ & $Z_{2}^{-} \times Z_{2}^{c}$ & $Z_{2}^{-}$ & & \\
$K_{5}$ & $Z_{2}$ & $Z_{2}^{-}$ & $I$ & & \\
$K_{6}, K_{12}$ & $Z_{4}$ & $Z_{2}$ & $Z_{2}^{-}$ & $I$ & \\
$K_{7}, K_{11}$ & $Z_{3} \times Z_{2}^{c}$ & $Z_{2}$ & $Z_{2}^{-}$ & $I$ & \\
$K_{8}$ & $D_{3}$ & $D_{2}$ & $Z_{2}^{c}$ & $I$ & & \\
$K_{9}, K_{15}$ & $D_{3}$ & $D_{4}$ & $D_{2}$ & $Z_{2}$ & $Z_{2}$ & $I$ \\
$K_{10}$ & $Z_{3}$ & $Z_{2}^{-}$ & $Z_{2}$ & $I$ & & \\
$K_{13}$ & $Z_{8}$ & $Z_{2}$ & $Z_{2}^{-}$ & $I$ & & \\
\hline
\end{tabular}

For $\mathrm{O}(2)$, we have $U^{\ell} \otimes U^{\ell}=U^{2 \ell} \oplus U^{0 g} \oplus U^{0 u}$ and $U^{\ell_{1}} \otimes U^{\ell_{2}}=U^{\ell_{1}+\ell_{2}} \oplus U^{\left|\ell_{1}-\ell_{2}\right|}$ for $\ell_{2} \neq \ell_{1}$.

If $K_{i}=H$, then the space $\mathrm{V}$ is spanned by the spaces of the copies of all trivial representations of $K_{i}$ that belong to $\mathrm{S}^{2}\left(\mathrm{~S}^{2}(g)\right)$. This gives us a method for calculation of the $\operatorname{dimension} \operatorname{dim} V$ alternative to that in [2]. Otherwise, it is spanned by the spaces of all irreducible representations of $K_{i}$ that contain at least one copy of the trivial representation of $H$. To determine such representations, we use Table $n .9$ from [1].

\subsection{The Basis of the Space $V^{H}$ for Different Groups}

We start from the basis for $K_{2}$. Gordienko [10] proposed a basis $\left\{\mathbf{h}_{\ell}^{m}:-\ell \leq m \leq \ell\right\}$ in the space of the irreducible representation $U^{\ell}$ of the group $\mathrm{SO}(3)$ in which all matrix entries of the representation's matrices become real-valued functions. Godunov and Cordienko [8] found the coefficients $g_{\ell\left[\ell_{1}, \ell_{2}\right]}^{m\left[m_{2}, m_{2}\right]}$ of the expansion

$$
\mathbf{h}_{\ell_{1}}^{m_{1}} \otimes \mathbf{h}_{\ell_{2}}^{m_{2}}=\sum_{\ell=\left|\ell_{1}-\ell_{2}\right|}^{\ell_{1}+\ell_{2}} \sum_{m=-\ell}^{\ell} g_{\ell\left[\ell_{1}, \ell_{2}\right]}^{m\left[m_{1}, m_{2}\right]} \mathbf{h}_{\ell}^{m} .
$$

We call them the Godunov-Gordienko coefficients. Malyarenko and Ostoja-Starzewski [22] calculated the tensors of the basis of the 21-dimensional space $S^{2}\left(\mathrm{~S}^{2}\left(\mathbb{R}^{3}\right)\right)$ for the group $K_{2}$ in terms of the above coefficients, see Table 3 in the electronic version of this paper [21]. Using MATLAB Symbolic Math Toolbox, we calculate the elements of the bases for the groups $K_{1}, K_{3}-K_{16}$ as linear combinations of the tensors of the basis for the group $K_{2}$, see [21, Table 3].

\subsection{The Isotropy Subgroups for the Groups $K_{i}$}

Table 3 shows the isotropy subgroups of the groups $K_{i}$. In this table, $Z_{2}^{-}$is the order 2 group generated by the reflection through the $y z$-plane, and $\tilde{Z}_{2}$ is the group generated by a reflection leavind an edge of a cube invariant [9]. The group $H_{0}$ is always equal to $K_{i}$ and therefore is omitted. 


\subsection{The Orbit Type Stratification}

The following formulae describe the orbit type stratification of the orbit space $\hat{\mathbb{R}}^{3} / K_{i}$. The zeroth stratum is always equal to $\{\mathbf{0}\}$ and therefore is omitted.

$\hat{\mathbb{R}}^{3} / K_{1}$ :

$$
\left(\hat{\mathbb{R}}^{3} / K_{1}\right)_{1}=\left\{p_{3}>0\right\} \cup\left\{p_{2}>0, p_{3}=0\right\} \cup\left\{\left(p_{1}, 0,0\right): p_{1}>0\right\} .
$$

$\hat{\mathbb{R}}^{3} / K_{2}, \hat{\mathbb{R}}^{3} / K_{16}$ :

$$
\left(\hat{\mathbb{R}}^{3} / K_{2}\right)_{1}=\left\{\left(0,0, p_{3}\right): p_{3}>0\right\} .
$$

$\hat{\mathbb{R}}^{3} / K_{3}$ :

$$
\begin{aligned}
& \left(\hat{\mathbb{R}}^{3} / K_{3}\right)_{1}=\left\{\left(0,0, p_{3}\right): p_{3}>0\right\}, \\
& \left(\hat{\mathbb{R}}^{3} / K_{3}\right)_{2}=\left\{\left(p_{1} \neq 0,0, p_{3}>0\right)\right\}, \\
& \left(\hat{\mathbb{R}}^{3} / K_{3}\right)_{3}=\left\{\left(p_{1}, p_{2}>0, p_{3}>0\right): p_{3}>0\right\} .
\end{aligned}
$$

$\hat{\mathbb{R}}^{3} / K_{4}, \hat{\mathbb{R}}^{3} / K_{14}$ :

$$
\begin{aligned}
& \left(\hat{\mathbb{R}}^{3} / K_{4}\right)_{1}=\left\{\left(p_{1}, 0,0\right): p_{1}>0\right\}, \\
& \left(\hat{\mathbb{R}}^{3} / K_{4}\right)_{2}=\left\{\left(0,0, p_{3}\right): p_{3}>0\right\}, \\
& \left(\hat{\mathbb{R}}^{3} / K_{4}\right)_{3}=\left\{\left(p_{1}, 0, p_{3}\right): p_{1}>0, p_{3}>0\right\} .
\end{aligned}
$$

$\hat{\mathbb{R}}^{3} / K_{5}$ :

$$
\begin{aligned}
& \left(\hat{\mathbb{R}}^{3} / K_{5}\right)_{1}=\{(\lambda, 0,0): \lambda>0\}, \\
& \left(\hat{\mathbb{R}}^{3} / K_{5}\right)_{2}=\left\{\left(\lambda, \theta_{p}, 0\right): \lambda>0,0<\theta_{p}<\pi / 2\right\}, \\
& \left(\hat{\mathbb{R}}^{3} / K_{5}\right)_{3}=\left\{\left(\lambda, \pi / 2, \varphi_{p}\right): \lambda>0,0<\varphi_{p}<\pi / m\right\}, \\
& \left(\hat{\mathbb{R}}^{3} / K_{5}\right)_{4}=\left\{\left(\lambda, \theta_{p}, \varphi_{p}\right): \lambda>0,0<\theta_{p}<\pi / 2,0<\varphi_{p}<\pi / m\right\}
\end{aligned}
$$

for $m=1$, where $\left(\lambda, \theta_{p}, \varphi_{p}\right)$ are the spherical coordinates in $\hat{\mathbb{R}}^{3}$.

$\hat{\mathbb{R}}^{3} / K_{6}, \hat{\mathbb{R}}^{3} / K_{12}:(17)$ with $m=2$.

$\hat{\mathbb{R}}^{3} / K_{7}, \hat{\mathbb{R}}^{3} / K_{11}$ : (17) with $m=3$.

$\hat{\mathbb{R}}^{3} / K_{8}$ :

$$
\begin{aligned}
& \left(\hat{\mathbb{R}}^{3} / K_{8}\right)_{1}=\{(\lambda, \pi / 4,0): \lambda>0\} \\
& \left(\hat{\mathbb{R}}^{3} / K_{8}\right)_{2}=\{(\lambda, 0,0): \lambda>0\} \\
& \left(\hat{\mathbb{R}}^{3} / K_{8}\right)_{3}=\left\{\left(\lambda, \theta_{p}, 0\right): \lambda>0,0<\theta_{p}<\pi / 4\right\} \\
& \left(\hat{\mathbb{R}}^{3} / K_{8}\right)_{3}=\left\{\left(\lambda, \theta_{p}, \varphi\right): \lambda>0,0<\varphi_{p}<\pi / 2,0<\theta_{p}<\cot ^{-1}\left(\sqrt{2} \cos \left(\varphi_{p}-\pi / 4\right)\right)\right\} .
\end{aligned}
$$


$\hat{\mathbb{R}}^{3} / K_{9}, \hat{\mathbb{R}}^{3} / K_{15}$ :

$$
\begin{aligned}
& \left(\hat{\mathbb{R}}^{3} / K_{9}\right)_{1}=\left\{\left(p_{1}, p_{2}, p_{3}\right): 0<p_{1}=p_{2}=p_{3}\right\}, \\
& \left(\hat{\mathbb{R}}^{3} / K_{9}\right)_{2}=\left\{\left(0,0, p_{3}\right): p_{3}>0\right\}, \\
& \left(\hat{\mathbb{R}}^{3} / K_{9}\right)_{3}=\left\{\left(0, p_{2}, p_{3}\right): 0<p_{2}=p_{3}\right\}, \\
& \left(\hat{\mathbb{R}}^{3} / K_{9}\right)_{4}=\left\{\left(0, p_{2}, p_{3}\right): 0<p_{2}<p_{3}\right\}, \\
& \left(\hat{\mathbb{R}}^{3} / K_{9}\right)_{5}=\left\{\left(p_{1}, p_{2}, p_{3}\right): 0<p_{1}=p_{2}<p_{3}\right\}, \\
& \left(\hat{\mathbb{R}}^{3} / K_{9}\right)_{6}=\left\{\left(p_{1}, p_{2}, p_{3}\right): 0<p_{1}<p_{2}<p_{3}\right\} .
\end{aligned}
$$

$\hat{\mathbb{R}}^{3} / K_{10}$ :

$$
\begin{aligned}
& \left(\hat{\mathbb{R}}^{3} / K_{10}\right)_{1}=\{(\lambda, 0,0): \lambda>0\}, \\
& \left(\hat{\mathbb{R}}^{3} / K_{10}\right)_{2}=\left\{\left(\lambda, \theta_{p}, 0\right): \lambda>0,0<\theta_{p}<\pi / 2\right\}, \\
& \left(\hat{\mathbb{R}}^{3} / K_{10}\right)_{3}=\left\{\left(\lambda, \pi / 2, \varphi_{p}\right): \lambda>0,0<\varphi_{p}<\pi / 3\right\}, \\
& \left(\hat{\mathbb{R}}^{3} / K_{10}\right)_{4}=\left\{\left(\lambda, \theta / 2, \varphi_{p}\right): \lambda>0,0<\theta_{p}<\pi / 3,0<\varphi_{p}<\pi / 3\right\} .
\end{aligned}
$$

$\hat{\mathbb{R}}^{3} / K_{13}:(17)$ with $m=4$.

\section{The Results}

In Theorem $m$ below we denote by ${ }_{K_{m}} \mathrm{~T}_{i j k l}$ the tensors of the basis given in [21, Table 3] in the lines marked by $K_{m}, 1 \leq m \leq 16$. We say "a triclinic (orthotropic, etc.) random field" instead of more rigourous "a random field with triclinic (orthotropic, etc.) symmetry". A sketch of proofs is given in [21, Section 6].

\subsection{The Triclinic Class}

Theorem 1 (A triclinic random field in the triclinic class) The one-point correlation tensor of a homogeneous and $\left(Z_{2}^{c}, 21 A_{g}\right)$-isotropic random field $\mathrm{C}(\mathbf{x})$ is

$$
\left\langle\left.\mathbf{C}(\mathbf{x})\right|_{i j k l}=\sum_{m=1}^{21} C_{m} Z_{2}^{c} T_{i j k l}^{A_{g}, m, 1}\right.
$$

where $C_{m} \in \mathbb{R}$. Its two-point correlation tensor has the form

$$
\langle\mathrm{C}(\mathbf{x}), \mathrm{C}(\mathbf{y})\rangle=\int_{\hat{\mathbb{R}}^{3} / Z_{2}^{c}} \cos (\mathbf{p}, \mathbf{y}-\mathbf{x}) f(\mathbf{p}) \mathrm{d} \Phi(\mathbf{p}),
$$

where $f(\mathbf{p})$ is a $\Phi$-equivalence class of measurable functions acting from $\hat{\mathbb{R}}^{3} / Z_{2}^{c}$ to the set of nonnegative-definite symmetric linear operators on $\mathrm{V}_{2}^{Z_{2}^{c}}$ with unit trace, and $\Phi$ is a finite 
measure on $\hat{\mathbb{R}}^{3} / Z_{2}^{c}$. The field has the form

$$
\begin{aligned}
\mathrm{C}_{i j k l}(\mathbf{x})= & \sum_{m=1}^{21} C_{m} Z_{2}^{c} \mathrm{~T}_{i j k l}^{A_{g}, m, 1}+\sum_{m=1}^{21} \int_{\hat{\mathbb{R}}^{3} / Z_{2}^{c}} \cos (\mathbf{p}, \mathbf{x}) \mathrm{d} Z_{m}^{1}(\mathbf{p})_{Z_{2}^{c}} \mathbf{T}_{i j k l}^{A_{g}, m, 1} \\
& +\sum_{m=1}^{21} \int_{\hat{\mathbb{R}}^{3} / Z_{2}^{c}} \sin (\mathbf{p}, \mathbf{x}) \mathrm{d} Z_{m}^{2}(\mathbf{p})_{Z_{2}^{c}} \mathbf{T}_{i j k l}^{A_{g}, m, 1}
\end{aligned}
$$

where $\left(Z_{1}^{m}(\mathbf{p}), \ldots, Z_{21}^{m}(\mathbf{p})\right)^{\top}$ are two centred uncorrelated $\mathrm{V}_{2}^{Z_{2}^{c}}$-valued random measures on $\hat{\mathbb{R}}^{3} / Z_{2}^{c}$ with control measure $f(\mathbf{p}) \mathrm{d} \Phi(\mathbf{p})$.

Theorem 2 (An isotropic random field in the triclinic class) The one-point correlation tensor of a homogeneous and $\left(\mathrm{O}(3), 2 U^{0 g} \oplus 2 U^{2 g} \oplus U^{4 g}\right)$-isotropic random field $\mathrm{C}(\mathbf{x})$ is

$$
\langle\mathrm{C}(\mathbf{x})\rangle_{i j k l}=C_{1} \mathrm{~T}_{i j k l}^{U^{0 g}, 1,1}+C_{2} \mathrm{~T}_{i j k l}^{U^{0 g}, 2,1},
$$

where $C_{1}, C_{2} \in \mathbb{R}$. Its two-point correlation tensor has the spectral expansion

$$
\left\langle\mathrm{C}(\mathbf{x}),\left.\mathrm{C}(\mathbf{y})\right|_{i j k l l^{\prime} j^{\prime} k^{\prime} l^{\prime}}=\sum_{n=1}^{3} \int_{0}^{\infty} \sum_{q=1}^{29} N_{n q}(\lambda, \rho) L_{i i k l l^{\prime} j^{\prime} k^{\prime} l^{\prime}}^{q}(\mathbf{y}-\mathbf{x}) \mathrm{d} \Phi_{n}(\lambda) .\right.
$$

The measures $\Phi_{n}(\lambda)$ satisfy the condition

$$
\Phi_{2}(\{0\})=2 \Phi_{3}(\{0\}) .
$$

The spectral expansion of the field has the form

$$
\begin{aligned}
\mathrm{C}_{i j k l}(\rho, \theta, \varphi)= & C_{1} \mathrm{~T}_{i j k l}^{U^{0 g}, 1,1}+C_{2} \mathrm{~T}_{i j k l}^{U^{0 g}, 2,1} \\
& +2 \sqrt{\pi} \sum_{m=1}^{13} \sum_{t=0}^{\infty} \sum_{u=-t}^{t} \int_{0}^{\infty} j_{t}(\lambda \rho) \mathrm{d} Z_{m t u i j k l}(\lambda) S_{t}^{u}(\theta, \varphi),
\end{aligned}
$$

where $S_{t}^{u}(\theta, \varphi)$ are real-valued spherical harmonics.

The functions $N_{n q}(\lambda, \rho)$ and $L_{i i k l i^{\prime} j^{\prime} k^{\prime} l^{\prime}}^{q}(\mathbf{y}-\mathbf{x})$ are given in [21, Tables 5, 6].

\subsection{The Monoclinic Class}

Theorem 3 (A monoclinic random field in the monoclinic class) The one-point correlation tensor of a homogeneous and $\left(Z_{2} \times Z_{2}^{c}, 13 A_{g}\right)$-isotropic random field $\mathrm{C}(\mathbf{x})$ is

$$
\langle\mathbf{C}(\mathbf{x})\rangle_{i j k l}=\sum_{m=1}^{13} C_{m} Z_{2} \times Z_{2}^{c} \mathbf{T}_{i j k l}^{A g, m, 1}
$$

where $C_{m} \in \mathbb{R}$. Its two-point correlation tensor has the form

$$
\langle\mathrm{C}(\mathbf{x}), \mathrm{C}(\mathbf{y})\rangle=\frac{1}{2} \int_{\hat{\mathbb{R}}^{3} / Z_{2} \times Z_{2}^{c}} \cos \left(p_{1}\left(y_{1}-x_{1}\right)+p_{2}\left(y_{2}-x_{2}\right)\right) \cos \left(p_{3}\left(y_{3}-x_{3}\right)\right) f(\mathbf{p}) \mathrm{d} \Phi(\mathbf{p}),
$$


where $f(\mathbf{p})$ is a $\Phi$-equivalence class of measurable functions acting from $\hat{\mathbb{R}}^{3} / Z_{2} \times Z_{2}^{c}$ to the set of nonnegative-definite symmetric linear operators on $\mathrm{V}_{2} \times Z_{2}^{c}$ with unit trace, and $\Phi$ is a finite Radon measure on $\hat{\mathbb{R}}^{3} / Z_{2} \times Z_{2}^{c}$. The field has the form

$$
\begin{aligned}
\mathrm{C}(\mathbf{x})_{i j k l}= & \sum_{m=1}^{13} C_{m} Z_{2} \times Z_{2}^{c} T_{i j k l}^{A_{g}, m, 1} \\
& +\frac{1}{\sqrt{2}} \sum_{m=1}^{13} \int_{\hat{\mathbb{R}}^{3} / Z_{2} \times Z_{2}^{c}} \cos \left(p_{1} x+p_{2} y\right) \cos \left(p_{3} z\right) \mathrm{d} \mathbf{Z}_{m}^{1}(\mathbf{p})_{Z_{2} \times Z_{2}^{c}} T_{i j k l}^{A_{g}, m, 1} \\
& +\frac{1}{\sqrt{2}} \sum_{m=1}^{13} \int_{\hat{\mathbb{R}}^{3} / Z_{2} \times Z_{2}^{c}} \sin \left(p_{1} x+p_{2} y\right) \sin \left(p_{3} z\right) \mathrm{d} Z_{m}^{2}(\mathbf{p})_{Z_{2} \times Z_{2}^{c}} \mathbf{T}_{i j k l}^{A_{g}, m, 1} \\
& +\frac{1}{\sqrt{2}} \sum_{m=1}^{13} \int_{\hat{\mathbb{R}}^{3} / Z_{2} \times Z_{2}^{c}} \cos \left(p_{1} x+p_{2} y\right) \sin \left(p_{3} z\right) \mathrm{d} Z_{m}^{3}(\mathbf{p})_{Z_{2} \times Z_{2}^{c}} T_{i j k l}^{A_{g}, m, 1} \\
& +\frac{1}{\sqrt{2}} \sum_{m=1}^{13} \int_{\hat{\mathbb{R}}^{3} / Z_{2} \times Z_{2}^{c}} \sin \left(p_{1} x+p_{2} y\right) \cos \left(p_{3} z\right) \mathrm{d} Z_{m}^{4}(\mathbf{p})_{Z_{2} \times Z_{2}^{c}} T_{i j k l}^{A_{g}, m, 1}
\end{aligned}
$$

where $\left(Z_{1}^{n}(\mathbf{p}), \ldots, Z_{13}^{n}(\mathbf{p})\right)^{\top}$ are four centred uncorrelated $\mathrm{V}^{Z_{2} \times Z_{2}^{c}}$-valued random measures on $\hat{\mathbb{R}}^{3} / Z_{2} \times Z_{2}^{c}$ with control measure $f(\mathbf{p}) \mathrm{d} \Phi(\mathbf{p})$.

Theorem 4 (A transverse isotropic random field in the monoclinic class) The one-point correlation tensor of a homogeneous and $\left(\mathrm{O}(2) \times Z_{2}^{c}, 5 U^{0 g g} \oplus 3 U^{2 g} \oplus U^{4 g}\right)$-isotropic random field $\mathrm{C}(\mathbf{x})$ is

$$
\langle\mathrm{C}(\mathbf{x})\rangle_{i j k l}=\sum_{m=1}^{5} C_{m} \mathrm{O}(2) \times Z_{2}^{c} \mathrm{~T}_{i j k l}^{U^{0 g g}, m, 1},
$$

where $C_{m} \in \mathbb{R}$. Its two-point correlation tensor has the form

$$
\langle\mathrm{C}(\mathbf{x}), \mathrm{C}(\mathbf{y})\rangle=\int_{\hat{\mathbb{R}}^{3} / \mathrm{O}(2) \times Z_{2}^{c}} J_{0}\left(\sqrt{\left(p_{1}^{2}+p_{2}^{2}\right)\left(z_{1}^{2}+z_{2}^{2}\right)}\right) \cos \left(p_{3} z_{3}\right) f(\mathbf{p}) \mathrm{d} \Phi(\mathbf{p}),
$$

where $\Phi$ is a measure on $\hat{\mathbb{R}}^{3} / \mathrm{O}(2) \times Z_{2}^{c}$, and $f(\mathbf{p})$ is a $\Phi$-equivalence class of measurable functions on $\hat{\mathbb{R}}^{3} / \mathrm{O}(2) \times Z_{2}^{c}$ with values in the compact set of all nonnegative-definite linear operators in the space $\mathrm{V}^{\mathrm{O}(2) \times Z_{2}^{c}}$ with unit trace of the form

$$
\left(\begin{array}{ccccc}
A & 0 & 0 & 0 & 0 \\
0 & B_{1} & B_{2} & B_{3} & 0 \\
0 & B_{2} & B_{4} & B_{5} & 0 \\
0 & B_{3} & B_{5} & B_{6} & 0 \\
0 & 0 & 0 & 0 & B_{7}
\end{array}\right)
$$


where $A$ is a nonnegative-definite $5 \times 5$ matrix, and $B_{m}, 1 \leq m \leq 7$ are $2 \times 2$ matrices proportional to the identity matrix. The field has the form

$$
\begin{aligned}
\mathrm{C}(\mathbf{x})= & \sum_{m=1}^{5} C_{m} \mathrm{~T}_{i j k l}^{m} \\
& +\sum_{m=1}^{13} \int_{\hat{\mathbb{R}}^{3} / \mathrm{O}(2) \times Z_{2}^{c}} J_{0}\left(\sqrt{\left(p_{1}^{2}+p_{2}^{2}\right)\left(z_{1}^{2}+z_{2}^{2}\right)}\right) \\
& \times\left(\cos \left(p_{3} z\right) \mathrm{d} Z_{m}^{01}(\mathbf{p}) \mathrm{T}_{i j k l}^{m}+\sin \left(p_{3} z\right) \mathrm{d} Z_{m}^{02}(\mathbf{p}) \mathrm{T}_{i j k l}^{m}\right) \\
& +\sqrt{2} \sum_{\ell=1}^{\infty} \sum_{m=1}^{13} \int_{\hat{\mathbb{R}}^{3} / \mathrm{O}(2) \times Z_{2}^{c}} J_{\ell}\left(\sqrt{\left(p_{1}^{2}+p_{2}^{2}\right)\left(z_{1}^{2}+z_{2}^{2}\right)}\right) \\
& \times\left(\cos \left(p_{3} z\right) \cos \left(\ell \varphi_{p}\right) \mathrm{d} Z_{m}^{\ell 1}(\mathbf{p}) \mathrm{T}_{i j k l}^{m}+\cos \left(p_{3} z\right) \sin \left(\ell \varphi_{p}\right) \mathrm{d} Z^{\ell 2 m}(\mathbf{p}) \mathrm{T}_{i j k l}^{m}\right. \\
& \left.+\sin \left(p_{3} z\right) \cos \left(\ell \varphi_{p}\right) \mathrm{d} Z_{m}^{\ell 3}(\mathbf{p}) \mathrm{T}_{i j k l}^{m}+\sin \left(p_{3} z\right) \sin \left(\ell \varphi_{p}\right) \mathrm{d} Z_{m}^{\ell 4}(\mathbf{p}) \mathrm{T}_{i j k l}^{m}\right)
\end{aligned}
$$

where $\left(Z_{1}^{\ell i}(\mathbf{p}), \ldots, Z_{13}^{\ell i}(\mathbf{p})\right)^{\top}$ are centred uncorrelated $\mathrm{V}^{\mathrm{O}(2) \times Z_{2}^{c}}$-valued random measures on $\hat{\mathbb{R}}^{3} / \mathrm{O}(2) \times Z_{2}^{c}$ with control measure $f(\mathbf{p}) \mathrm{d} \Phi(\mathbf{p})$, and

$$
\mathrm{T}_{i j k l}^{m}= \begin{cases}\mathrm{O}(2) \times Z_{2}^{c} \mathrm{~T}^{U^{0 g g}, m, 1}, & \text { if } 1 \leq m \leq 5, \\ \mathrm{O}(2) \times Z_{2}^{c} \mathrm{~T}^{U^{2 g},\lfloor m / 2\rfloor-2, m \bmod 2+1}, & \text { if } 6 \leq m \leq 11, \\ \mathrm{O}(2) \times Z_{2}^{c} \mathrm{~T}^{U^{4 g}, 1, m-11}, & \text { if } 12 \leq m \leq 13\end{cases}
$$

\subsection{The Orthotropic Class}

Theorem 5 (An orthotropic random field in the orthotropic class) The one-point correlation tensor of a homogeneous and $\left(D_{2} \times Z_{2}^{c}, 9 A_{g}\right)$-isotropic random field $\mathrm{C}(\mathbf{x})$ is

$$
\langle\mathbf{C}(\mathbf{x})\rangle_{i j k l}=\sum_{m=1}^{9} C_{m D_{2} \times Z_{2}^{c}} \mathbf{T}_{i j k l}^{A_{g}, m, 1},
$$

where $C_{m} \in \mathbb{R}$. Its two-point correlation tensor has the form

$$
\langle\mathbf{C}(\mathbf{x}), \mathbf{C}(\mathbf{y})\rangle=\int_{\hat{\mathbb{R}}^{3} / D_{2} \times Z_{2}^{c}} \cos \left(p_{1}\left(y_{1}-x_{1}\right)\right) \cos \left(p_{2}\left(y_{2}-x_{2}\right)\right) \cos \left(p_{3}\left(y_{3}-x_{3}\right)\right) f(\mathbf{p}) \mathrm{d} \Phi(\mathbf{p}),
$$

where $f(\mathbf{p})$ is a $\Phi$-equivalence class of measurable functions acting from $\hat{\mathbb{R}}^{3} / D_{2} \times Z_{2}^{c}$ to the set of nonnegative-definite symmetric linear operators on $\mathrm{V}^{D_{2}} \times Z_{2}^{c}$ with unit trace, and $\Phi$ is a finite measure on $\hat{\mathbb{R}}^{3} / D_{2} \times Z_{2}^{c}$. The field has the form

$$
\mathrm{C}(\mathbf{x})_{i j k l}=\sum_{m=1}^{9} C_{m D_{2} \times Z_{2}^{c}} \mathbf{T}_{i j k l}^{A_{g}, m, 1}+\sum_{m=1}^{9} \sum_{n=1}^{8} \int_{\hat{\mathbb{R}}^{3} / D_{2} \times Z_{2}^{c}} u_{n}(\mathbf{p}, \mathbf{x}) \mathrm{d} Z_{m}^{n}(\mathbf{p})_{D_{2} \times Z_{2}^{c}} \mathbf{T}_{i j k l}^{A_{g}, m, 1},
$$

where $\left(Z_{1}^{n}(\mathbf{p}), \ldots, Z_{9}^{n}(\mathbf{p})\right)^{\top}$ are eight centred uncorrelated $\mathrm{V}^{D_{2} \times Z_{2}^{c}}$-valued random measures on $\hat{\mathbb{R}}^{3} / D_{2} \times Z_{2}^{c}$ with control measure $f(\mathbf{p}) \mathrm{d} \Phi(\mathbf{p})$, and where $u_{n}(\mathbf{p}, \mathbf{x})$ are eight different product of sines and cosines of $p_{r} x_{r}$. 
Consider a $9 \times 9$ symmetric nonnegative-definite matrix with the unit trace of the following structure:

$$
\left(\begin{array}{cc}
A & B \\
B^{\top} & C
\end{array}\right)
$$

where $A$ is a $6 \times 6$ matrix. Introduce the following notation:

$$
\begin{aligned}
& j_{1}(\mathbf{p}, \mathbf{z})=\cos \left(p_{1} z_{1}\right) \cos \left(p_{2} z_{2}\right) \cos \left(p_{3} z_{3}\right), \\
& j_{2}(\mathbf{p}, \mathbf{z})=\cos \left(p_{1} z_{2}\right) \cos \left(p_{2} z_{1}\right) \cos \left(p_{3} z_{3}\right) .
\end{aligned}
$$

Let $\Phi$ be a finite measure on $\hat{\mathbb{R}}^{3} / D_{4} \times Z_{2}^{c}$. Let $f^{0}(\mathbf{p})$ be a $\Phi$-equivalence class of measurable functions acting from $\left(\hat{\mathbb{R}}^{3} / D_{4} \times Z_{2}^{c}\right)_{m}, 0 \leq m \leq 1$ to the set of nonnegative-definite symmetric matrices with unit trace satisfying $B=0$. Let $f^{+}(\mathbf{p})$ be a $\Phi$-equivalence class of measurable functions acting from $\left(\hat{\mathbb{R}}^{3} / D_{4} \times Z_{2}^{c}\right)_{m}, 2 \leq m \leq 4$ to the set of nonnegativedefinite symmetric linear operators on $\mathrm{V}^{D_{4} \times Z_{2}^{c}}$ with unit trace, and let $f^{-}(\mathbf{p})$ is obtained from $f^{+}(\mathbf{p})$ by multiplying $B$ and $B^{\top}$ by -1 .

Theorem 6 (A tetragonal random field in the orthotropic class) The one-point correlation tensor of a homogeneous and $\left(D_{4} \times Z_{2}^{c}, 6 A_{1 g} \oplus 3 B_{1 g}\right)$-isotropic random field $\mathrm{C}(\mathbf{x})$ is

$$
\langle\mathrm{C}(\mathbf{x})\rangle_{i j k l}=\sum_{m=1}^{6} C_{m D_{4} \times Z_{2}^{c}} \mathrm{~T}_{i j k l}^{A_{g 1}, m, 1},
$$

where $C_{m} \in \mathbb{R}$. Its two-point correlation tensor has the form

$$
\begin{aligned}
\langle\mathrm{C}(\mathbf{x}), \mathrm{C}(\mathbf{y})\rangle= & \frac{1}{2} \sum_{m=0}^{1} \int_{\left(\hat{\mathbb{R}}^{3} / D_{4} \times Z_{2}^{c}\right) m}\left[j_{1}(\mathbf{p}, \mathbf{y}-\mathbf{x})+j_{2}(\mathbf{p}, \mathbf{y}-\mathbf{x})\right] f^{0}(\mathbf{p}) \mathrm{d} \Phi(\mathbf{p}) \\
& +\frac{1}{2} \sum_{m=2}^{4} \int_{\left(\hat{\mathbb{R}}^{3} / D_{4} \times Z_{2}^{c}\right)_{m}}\left[j_{1}(\mathbf{p}, \mathbf{y}-\mathbf{x}) f^{+}(\mathbf{p})+j_{2}(\mathbf{p}, \mathbf{y}-\mathbf{x}) f^{-}(\mathbf{p})\right] \mathrm{d} \Phi(\mathbf{p}) .
\end{aligned}
$$

The field has the form

$$
\begin{aligned}
\mathrm{C}(\mathbf{x})_{i j k l}= & \sum_{m=1}^{6} C_{m} D_{4} \times Z_{2}^{c} \top_{i j k l}^{A_{1 g}, m, 1} \\
& +\frac{1}{\sqrt{2}} \sum_{q=1}^{9} \sum_{n=1}^{16} \sum_{m=0}^{1} \int_{\left(\hat{\mathbb{R}}^{3} / D_{4} \times Z_{2}^{c}\right)_{m}} u_{n}(\mathbf{p}, \mathbf{x}) \mathrm{d} Z_{q}^{n 0}(\mathbf{p})_{D_{4} \times Z_{2}^{c}} \mathrm{~T}_{i j k l}^{q} \\
& +\frac{1}{\sqrt{2}} \sum_{q=1}^{9} \sum_{n=1}^{8} \sum_{m=2}^{4} \int_{\left(\hat{\mathbb{R}}^{3} / D_{4} \times Z_{2}^{c}\right)_{m}} u_{n}(\mathbf{p}, \mathbf{x}) \mathrm{d} Z_{q}^{n+}(\mathbf{p})_{D_{4} \times Z_{2}^{c}} \mathrm{~T}_{i j k l}^{q} \\
& +\frac{1}{\sqrt{2}} \sum_{q=1}^{9} \sum_{n=9}^{16} \sum_{m=2}^{4} \int_{\left(\hat{\mathbb{R}}^{3} / D_{4} \times Z_{2}^{c}\right)_{m}} u_{n}(\mathbf{p}, \mathbf{x}) \mathrm{d} Z_{q}^{n-}(\mathbf{p})_{D_{4} \times Z_{2}^{c}} T_{i j k l}^{q},
\end{aligned}
$$

where $\left(Z_{1}^{n 0}(\mathbf{p}), \ldots, Z_{9}^{n 0}(\mathbf{p})\right)^{\top}\left(\operatorname{resp} .\left(Z_{1}^{n+}(\mathbf{p}), \ldots, Z_{9}^{n+}(\mathbf{p})\right)^{\top}\right.$, resp. $\left.\left(Z_{1}^{n-}(\mathbf{p}), \ldots, Z_{9}^{n-}(\mathbf{p})\right)^{\top}\right)$ are centred uncorrelated $\mathrm{V}^{D_{4} \times Z_{2}^{c}}$-valued random measures on the spaces $\left(\hat{\mathbb{R}}^{3} / D_{4} \times Z_{2}^{c}\right)_{m}$, 
Table 4 The functions $j_{n}(\mathbf{p}, \mathbf{z})$ for the tetrahedral group

\begin{tabular}{ll}
\hline$n$ & $j_{n}(\mathbf{p}, \mathbf{z})$ \\
\hline 1 & {$\left[\cos \left(p_{1} z_{1}+p_{2} z_{2}\right)+\cos \left(p_{1} z_{2}+p_{2} z_{1}\right)\right] \cos \left(p_{3} z_{3}\right)$} \\
2 & $\cos \left[\frac{1}{2}\left(\left(p_{1}+\sqrt{2} p_{3}\right) z_{1}+p_{2} z_{2}+\sqrt{2} p_{1} z_{3}\right)\right] \cos \left[\frac{1}{2}\left(p_{2} z_{1}+\left(-p_{1}+\sqrt{2} p_{3}\right) z_{2}-\sqrt{2} p_{2} z_{3}\right)\right]$ \\
& $+\cos \left[\frac{1}{2}\left(z_{1}\left(p_{1}-\sqrt{2} p_{3}\right)-p_{2} z_{2}-\sqrt{2} p_{1} z_{3}\right)\right] \cos \left[\frac{1}{2}\left(p_{2} z_{1}+\left(-p_{1}-\sqrt{2} p_{3}\right) z_{2}+\sqrt{2} p_{2} z_{3}\right)\right]$ \\
3 & $\cos \left[\frac{1}{2}\left(-p_{1} z_{1}+\left(p_{2}+\sqrt{2} p_{3}\right) z_{2}+\sqrt{2} p_{2} z_{3}\right)\right] \cos \left[\frac{1}{2}\left(\left(p_{2}-\sqrt{2} p_{3}\right) z_{1}-p_{1} z_{2}+\sqrt{2} p_{1} z_{3}\right)\right]$ \\
& $+\cos \left[\frac{1}{2}\left(-p_{1} z_{1}+\left(p_{2}-\sqrt{2} p_{3}\right) z_{2}-\sqrt{2} p_{2} z_{3}\right)\right] \cos \left[\frac{1}{2}\left(\left(p_{2}+\sqrt{2} p_{3}\right) z_{1}-p_{1} z_{2}-\sqrt{2} p_{1} z_{3}\right)\right]$ \\
\hline
\end{tabular}

$0 \leq m \leq 1$ (resp. $2 \leq m \leq 4)$ with control measure $f^{0}(\mathbf{p}) \mathrm{d} \Phi(\mathbf{p})$ (resp. $f^{+}(\mathbf{p}) \mathrm{d} \Phi(\mathbf{p})$, resp. $\left.f^{-}(\mathbf{p}) \mathrm{d} \Phi(\mathbf{p})\right), u_{n}(\mathbf{p}, \mathbf{x})$ are different product of sines and cosines of $p_{r} x_{r}$ for $1 \leq n \leq 8$ and eight different product of sines and cosines of $p_{1} x_{2}, p_{2} x_{1}$, and $p_{3} x_{3}$ for $9 \leq n \leq 16$, and

$$
\mathrm{T}_{i j k l}^{q}= \begin{cases}\mathrm{T}_{i j k l}^{A_{1 g}, q, 1}, & \text { if } 1 \leq q \leq 6, \\ \mathrm{~T}_{i j k l}^{B_{1 g}, q-6,1}, & \text { otherwise. }\end{cases}
$$

Consider a $9 \times 9$ symmetric nonnegative-definite matrix with unit trace of the following structure

$$
\left(\begin{array}{cccccc}
* & * & * & \mathbf{c}_{1}^{\top} & \mathbf{c}_{2}^{\top} & \mathbf{c}_{3}^{\top} \\
* & * & * & \mathbf{c}_{4}^{\top} & \mathbf{c}_{5}^{\top} & \mathbf{c}_{6}^{\top} \\
* & * & * & \mathbf{c}_{7}^{\top} & \mathbf{c}_{8}^{\top} & \mathbf{c}_{9}^{\top} \\
\mathbf{c}_{1} & \mathbf{c}_{4} & \mathbf{c}_{7} & A_{1} & A_{2} & A_{3} \\
\mathbf{c}_{2} & \mathbf{c}_{5} & \mathbf{c}_{8} & A_{2} & A_{4} & A_{5} \\
\mathbf{c}_{3} & \mathbf{c}_{6} & \mathbf{c}_{9} & A_{3} & A_{5} & A_{6}
\end{array}\right),
$$

where stars are arbitrary numbers, $\mathbf{c}_{i}$ are vectors with two components, and $A_{i}$ are $2 \times 2$ matrices. Let $\Phi$ be a finite measure on $\hat{\mathbb{R}}^{3} / \mathscr{T} \times Z_{2}^{c}$. Let $f^{0}(\mathbf{p})$ be a $\Phi$-equivalence class of measurable functions acting from $\left(\hat{\mathbb{R}}^{3} / \mathscr{T} \times Z_{2}^{c}\right)_{m}, 0 \leq m \leq 1$ to the set of nonnegativedefinite symmetric linear operators on $\mathrm{V}^{\mathscr{T} \times Z_{2}^{c}}$ with unit trace such that $\mathbf{c}_{i}=\mathbf{0}$ and $A_{i}$ are proportional to the identity matrix. Let $f^{1}(\mathbf{p})$ be a $\Phi$-equivalence class of measurable functions acting from $\left(\hat{\mathbb{R}}^{3} / \mathscr{T} \times Z_{2}^{c}\right)_{m}, 2 \leq m \leq 4$ to the set of nonnegative-definite symmetric linear operators on $\mathrm{V}^{\mathscr{T}} \times Z_{2}^{c}$ with unit trace. Denote

$$
g=\frac{1}{2}\left(\begin{array}{cc}
-1 & \sqrt{3} \\
-\sqrt{3} & -1
\end{array}\right) .
$$

Let $f^{+}(\mathbf{p})$ (resp. $f^{-}(\mathbf{p})$ ) is obtained from $f^{1}(\mathbf{p})$ by replacing all $\mathbf{c}_{i}$ with $g \mathbf{c}_{1}$ (resp. with $g^{-1} \mathbf{c}_{i}$ ) and all $A_{i}$ with $g A_{i} g^{-1}$ (resp. $g^{-1} A_{i} g$ ). Finally, let $j_{m}(\mathbf{p}, \mathbf{z})$ be functions from Table 4 .

Theorem 7 (A cubic random field in the orthotropic class) The one-point correlation tensor of a homogeneous and $\left(\mathscr{T} \times Z_{2}^{c}, 3 A_{g} \oplus 3\left({ }^{1} E_{g} \oplus{ }^{2} E_{g}\right)\right)$-isotropic random field $\mathrm{C}(\mathbf{x})$ is

$$
\langle\mathbf{C}(\mathbf{x})\rangle_{i j k l}=\sum_{m=1}^{3} C_{m} \mathscr{T} \times Z_{2}^{c} \mathbf{T}_{i j k l}^{A_{g}, m, 1},
$$


where $C_{m} \in \mathbb{R}$. Its two-point correlation tensor has the form

$$
\begin{aligned}
\langle\mathrm{C}(\mathbf{x}), \mathrm{C}(\mathbf{y})\rangle= & \frac{1}{6} \sum_{m=0}^{1} \int_{\left(\hat{\mathbb{R}}^{3} / \mathscr{T} \times Z_{2}^{c}\right)_{m}} \sum_{n=1}^{3} j_{n}(\mathbf{p}, \mathbf{y}-\mathbf{x}) f^{0}(\mathbf{p}) \mathrm{d} \Phi(\mathbf{p}) \\
& +\frac{1}{6} \sum_{m=2}^{4} \int_{\left(\hat{\mathbb{R}}^{3} / \mathscr{T} \times Z_{2}^{c}\right)_{m}}\left[j_{1}(\mathbf{p}, \mathbf{y}-\mathbf{x}) f^{1}(\mathbf{p})+j_{2}(\mathbf{p}, \mathbf{y}-\mathbf{x}) f^{+}(\mathbf{p})\right. \\
& \left.+j_{3}(\mathbf{p}, \mathbf{y}-\mathbf{x}) f^{-}(\mathbf{p})\right] \mathrm{d} \Phi(\mathbf{p}) .
\end{aligned}
$$

The field has the form

$$
\begin{aligned}
\mathrm{C}(\mathbf{x})= & \sum_{m=1}^{3} C_{m} \mathscr{T} \times Z_{2}^{c} \mathrm{~T}_{i j k l}^{A_{g}, m, 1}+\frac{1}{\sqrt{6}}\left(\sum_{q=1}^{9} \sum_{n=1}^{24} \sum_{m=0}^{1} \int_{\left(\hat{\mathbb{R}}^{3} / \mathscr{T} \times Z_{2}^{c}\right)_{m}} u_{n}(\mathbf{p}, \mathbf{x}) \mathrm{d} Z_{q}^{n 0}(\mathbf{p}) \mathrm{T}_{i j k l}^{q}\right. \\
& +\sum_{q=1}^{9} \sum_{n=1}^{8} \sum_{m=2}^{4} \int_{\left(\hat{\mathbb{R}}^{3} / \mathscr{T} \times Z_{2}^{c}\right)_{m}} u_{n}(\mathbf{p}, \mathbf{x}) \mathrm{d} Z_{q}^{n 1}(\mathbf{p}) \mathrm{T}_{i j k l}^{q} \\
& +\sum_{q=1}^{9} \sum_{n=9}^{16} \sum_{m=2}^{4} \int_{\left(\hat{\mathbb{R}}^{3} / \mathscr{T} \times Z_{2}^{c}\right)_{m}} u_{n}(\mathbf{p}, \mathbf{x}) \mathrm{d} Z_{q}^{n+}(\mathbf{p}) \mathrm{T}_{i j k l}^{q} \\
& \left.+\sum_{q=1}^{9} \sum_{n=17}^{24} \sum_{m=2}^{4} \int_{\left(\hat{\mathbb{R}}^{3} / \mathscr{T} \times Z_{2}^{c}\right)_{m}} u_{n}(\mathbf{p}, \mathbf{x}) \mathrm{d} Z_{q}^{n-}(\mathbf{p}) \mathrm{T}_{i j k l}^{q}\right),
\end{aligned}
$$

where $u_{n}(\mathbf{p}, \mathbf{x})$ are various products of sines and cosines of angles from Table 4 ,

$$
\mathrm{T}_{i j k l}^{q}= \begin{cases}\mathscr{T} \times Z_{2}^{c} \mathrm{~T}_{i j k l}^{A_{g}, q, 1}, & \text { if } 1 \leq q \leq 3 \\ \mathscr{T} \times Z_{2}^{c} \mathrm{~T}_{i j k l}^{E^{2 g},\lfloor q / 2\rfloor-1, q \bmod 2+1}, & \text { otherwise, }\end{cases}
$$

and where $\left(Z_{1}^{n 0}(\mathbf{p}), \ldots, Z_{9}^{n 0}(\mathbf{p})\right)^{\top}$ (resp. $\left(Z_{1}^{n 1}(\mathbf{p}), \ldots, Z_{9}^{n 1}(\mathbf{p})\right)^{\top}$, resp. $\left(Z_{1}^{n+}(\mathbf{p}), \ldots\right.$, $\left.Z_{9}^{n+}(\mathbf{p})\right)^{\top}$ resp. $\left.\left(Z_{1}^{n-}(\mathbf{p}), \ldots, Z_{9}^{n-}(\mathbf{p})\right)^{\top}\right)$ are centred uncorrelated $\mathrm{V}^{\mathscr{T} \times Z_{2}^{c}}$-valued random measures on $\left(\hat{\mathbb{R}}^{3} / \mathscr{T} \times Z_{2}^{c}\right)_{m}$ for $0 \leq m \leq 1$ (resp. $2 \leq m \leq 4$ ) with control measure $f^{0}(\mathbf{p}) \mathrm{d} \Phi(\mathbf{p})\left(\right.$ resp. $f^{1}(\mathbf{p}) \mathrm{d} \Phi(\mathbf{p})$, resp. $f^{+}(\mathbf{p}) \mathrm{d} \Phi(\mathbf{p})$, resp. $\left.f^{-}(\mathbf{p}) \mathrm{d} \Phi(\mathbf{p})\right)$.

Consider a $9 \times 9$ symmetric nonnegative-definite matrix with unit trace of the following structure

$$
\left(\begin{array}{lllllll}
* & * & * & * & * & \mathbf{c}_{1}^{\top} & \mathbf{c}_{2}^{\top} \\
* & * & * & * & * & \mathbf{c}_{3}^{\top} & \mathbf{c}_{4}^{\top} \\
* & * & * & * & * & \mathbf{c}_{5}^{\top} & \mathbf{c}_{6}^{\top} \\
* & * & * & * & * & \mathbf{c}_{7}^{\top} & \mathbf{c}_{8}^{\top} \\
* & * & * & * & * & \mathbf{c}_{9}^{\top} & \mathbf{c}_{10}^{\top} \\
\mathbf{c}_{1} & \mathbf{c}_{3} & \mathbf{c}_{5} & \mathbf{c}_{7} & \mathbf{c}_{9} & A_{1} & A_{2} \\
\mathbf{c}_{2} & \mathbf{c}_{4} & \mathbf{c}_{6} & \mathbf{c}_{8} & \mathbf{c}_{10} & A_{2} & A_{3}
\end{array}\right),
$$


Table 5 The matrices $g_{n}$ and the functions $j_{n}(\mathbf{p}, \mathbf{z})$ for the group $D_{6} \times Z_{2}^{c}$

\begin{tabular}{lll}
\hline$n$ & $g_{n}$ & $j_{n}(\mathbf{p}, \mathbf{z})$ \\
\hline 1 & $\left(\begin{array}{cc}1 & 0 \\
0 & 1\end{array}\right)$ & $\cos \left(p_{3} z_{3}\right) \cos \left(p_{1} z_{1}+p_{2} z_{2}\right)$ \\
2 & $\left(\begin{array}{cc}-1 & 0 \\
0 & 1\end{array}\right)$ & $\cos \left(p_{3} z_{3}\right) \cos \left(p_{1} z_{1}-p_{2} z_{2}\right)$ \\
3 & $\frac{1}{2}\left(\begin{array}{cc}1 & -\sqrt{3} \\
\sqrt{3} & 1\end{array}\right)$ & $\cos \left(p_{3} z_{3}\right) \cos \left[\left(p_{1}+\sqrt{3} p_{2}\right) z_{1}+\left(-\sqrt{3} p_{1}+p_{2}\right) z_{2}\right] / 2$ \\
4 & $\frac{1}{2}\left(\begin{array}{cc}-1 & \sqrt{3} \\
\sqrt{3} & -1\end{array}\right)$ & $\cos \left(p_{3} z_{3}\right) \cos \left[\left(p_{1}-\sqrt{3} p_{2}\right) z_{1}+\left(\sqrt{3} p_{1}+p_{2}\right) z_{2}\right] / 2$ \\
5 & $\frac{1}{2}\left(\begin{array}{cc}1 & \sqrt{3} \\
\sqrt{3} & -1\end{array}\right)$ & $\cos \left(p_{3} z_{3}\right) \cos \left[\left(-p_{1}+\sqrt{3} p_{2}\right) z_{1}+\left(\sqrt{3} p_{1}+p_{2}\right) z_{2}\right] / 2$ \\
6 & $\frac{1}{2}\left(\begin{array}{cc}1 & -\sqrt{3} \\
-\sqrt{3} & -1\end{array}\right)$ & $\cos \left(p_{3} z_{3}\right) \cos \left[\left(p_{1}+\sqrt{3} p_{2}\right) z_{1}+\left(\sqrt{3} p_{1}-p_{2}\right) z_{2}\right] / 2$ \\
\hline
\end{tabular}

where stars are arbitrary numbers, $\mathbf{c}_{i}$ are vectors with two components, and $A_{i}$ are $2 \times 2$ matrices of the form

$$
A_{j}=\left(\begin{array}{ll}
a-b & c+d \\
c-d & a+b
\end{array}\right)
$$

Let $\Phi$ be a finite measure on $\hat{\mathbb{R}}^{3} / D_{6} \times Z_{2}^{c}$. Let $f^{0}(\mathbf{p})$ be a $\Phi$-equivalence class of measurable functions acting from $\left(\hat{\mathbb{R}}^{3} / D_{6} \times Z_{2}^{c}\right)_{m}, 0 \leq m \leq 1$ to the set of nonnegative-definite symmetric matrices with unit trace such that $\mathbf{c}_{i}=\mathbf{0}$ and $A_{i}$ are proportional to the identity matrix. Let $f^{-}(\mathbf{p})$ be a $\Phi$-equivalence class of measurable functions acting from $\left(\hat{\mathbb{R}}^{3} / D_{6} \times Z_{2}^{c}\right)_{2}$ to the set of nonnegative-definite symmetric matrices with unit trace such that $A_{i}$ are symmetric. Let $f^{+}(\mathbf{p})$ be a $\Phi$-equivalence class of measurable functions acting from $\left(\hat{\mathbb{R}}^{3} / D_{6} \times Z_{2}^{c}\right)_{m}$, $3 \leq m \leq 4$ to the set of nonnegative-definite symmetric matrices with unit trace. Consider matrices and functions of Table 5 .

Let $f^{-i}(\mathbf{p})$ is obtained from $f^{-}(\mathbf{p})$ by replacing all $\mathbf{c}_{j}$ with $g_{i} \mathbf{c}_{j}$ and the vectors $(b, c)^{\top}$ in all $A_{j}$ with $g_{i}(b, c)^{\top}$. Let $f^{+i}(\mathbf{p})$ is obtained from $f^{+}(\mathbf{p})$ by replacing all $\mathbf{c}_{j}$ with $g_{i} \mathbf{c}_{j}$ and all $A_{j}$ with $g_{i} A_{j} g_{i}^{-1}$.

Theorem 8 (A hexagonal random field in the orthotropic class) The one-point correlation tensor of a homogeneous and $\left(D_{6} \times Z_{2}^{c}, 5 A_{1 g} \oplus 2 E_{2 g}\right)$-isotropic random field $\mathrm{C}(\mathbf{x})$ is

$$
\langle\mathrm{C}(\mathbf{x})\rangle_{i j k l}=\sum_{m=1}^{5} C_{m} D_{6} \times Z_{2}^{c} \mathbf{T}_{i j k l}^{A_{1 g}, m, 1},
$$

where $C_{m} \in \mathbb{R}$. Its two-point correlation tensor has the form

$$
\begin{aligned}
\langle\mathrm{C}(\mathbf{x}), \mathrm{C}(\mathbf{y})\rangle= & \frac{1}{6}\left(\sum_{m=0}^{1} \int_{\left(\hat{\mathbb{R}}^{3} / D_{6} \times Z_{2}^{c}\right)_{m}} \sum_{n=1}^{6} j_{n}(\mathbf{p}, \mathbf{y}-\mathbf{x}) f^{0}(\mathbf{p}) \mathrm{d} \Phi(\mathbf{p})\right. \\
& +\int_{\left(\hat{\mathbb{R}}^{3} / D_{6} \times Z_{2}^{c}\right)_{2}} \sum_{n=1}^{6} j_{n}(\mathbf{p}, \mathbf{y}-\mathbf{x}) f^{-n}(\mathbf{p}) \mathrm{d} \Phi(\mathbf{p}) \\
& \left.+\sum_{m=3}^{4} \int_{\left(\hat{\mathbb{R}}^{3} / D_{6} \times Z_{2}^{c}\right)_{m}} \sum_{n=1}^{6} j_{n}(\mathbf{p}, \mathbf{y}-\mathbf{x}) f^{+n}(\mathbf{p}) \mathrm{d} \Phi(\mathbf{p})\right) .
\end{aligned}
$$


The field has the form

$$
\begin{aligned}
\mathrm{C}(\mathbf{x})_{i j k l}= & \sum_{m=1}^{5} C_{m} D_{6} \times Z_{2}^{c} T_{i j k l}^{A_{1 g}, m, 1}+\frac{1}{\sqrt{6}} \sum_{q=1}^{9} \sum_{n=1}^{24} \sum_{m=0}^{1} \int_{\left(\hat{\mathbb{R}}^{3} / D_{6} \times Z_{2}^{c}\right)_{m}} u_{n}(\mathbf{p}, \mathbf{x}) \mathrm{d} Z_{q}^{0 n}(\mathbf{p})_{D_{6} \times Z_{2}^{c}} T_{i j k l}^{q} \\
& +\frac{1}{\sqrt{6}} \sum_{q=1}^{9} \sum_{s=1}^{6} \sum_{n=4 s-3}^{4 s} \int_{\left(\hat{\mathbb{R}}^{3} / D_{6} \times Z_{2}^{c}\right)_{2}} u_{n}(\mathbf{p}, \mathbf{x}) \mathrm{d} Z_{q}^{-n s}(\mathbf{p})_{D_{6} \times Z_{2}^{c}} T_{i j k l}^{q} \\
& +\frac{1}{\sqrt{6}} \sum_{q=1}^{9} \sum_{s=1}^{6} \sum_{n=4 s-3}^{4 s} \sum_{m=3}^{4} \int_{\left(\hat{\mathbb{R}}^{3} / D_{6} \times Z_{2}^{c}\right)_{m}} u_{n}(\mathbf{p}, \mathbf{x}) \mathrm{d} Z_{q}^{+n s}(\mathbf{p})_{D_{6} \times Z_{2}^{c}} T_{i j k l}^{q},
\end{aligned}
$$

where $\left(Z_{1}^{0 n}(\mathbf{p}), \ldots, Z_{9}^{0 n}(\mathbf{p})\right)^{\top} \quad\left(\right.$ resp. $\quad\left(Z_{1}^{-n s}(\mathbf{p}), \ldots, Z_{9}^{-n s}(\mathbf{p})\right)^{\top}, \quad$ resp. $\quad\left(Z_{1}^{+n s}(\mathbf{p}), \ldots\right.$, $\left.\left.Z_{9}^{+n s}(\mathbf{p})\right)^{\top}\right)$ are centred uncorrelated $\mathrm{V}^{D_{6} \times Z_{2}^{c}}$-valued random measures on $\left(\hat{\mathbb{R}}^{3} / D_{6} \times Z_{2}^{c}\right)_{m}$, $0 \leq m \leq 1$ (resp. on $\left(\hat{\mathbb{R}}^{3} / D_{6} \times Z_{2}^{c}\right)_{2}$, resp. on $\left.\left(\hat{\mathbb{R}}^{3} / D_{6} \times Z_{2}^{c}\right)_{2}, 3 \leq m \leq 4\right)$ with control measure $f^{0}(\mathbf{p}) \mathrm{d} \Phi(\mathbf{p})$ (resp. $f^{-s}(\mathbf{p}) \mathrm{d} \Phi(\mathbf{p})$, resp. $\left.f^{+s}(\mathbf{p}) \mathrm{d} \Phi(\mathbf{p})\right), u_{n}(\mathbf{p}, \mathbf{x}), 1 \leq n \leq 8$ are different product of sines and cosines of angles in Table 5, and where

$$
\mathrm{T}_{i j k l}^{q}= \begin{cases}D_{6} \times Z_{2}^{c} \mathrm{~T}_{i j k l}^{A_{g}, q, 1}, & \text { if } 1 \leq q \leq 5 \\ D_{6} \times Z_{2}^{c} \mathrm{~T}_{i j k l}^{E^{2 g},\lfloor q / 2\rfloor-1, q \bmod 2+1}, & \text { otherwise. }\end{cases}
$$

Consider a $9 \times 9$ symmetric nonnegative-definite matrix with unit trace of the following structure

$$
\left(\begin{array}{llllll}
* & * & * & \mathbf{c}_{1}^{\top} & \mathbf{c}_{2}^{\top} & \mathbf{c}_{3}^{\top} \\
* & * & * & \mathbf{c}_{4}^{\top} & \mathbf{c}_{5}^{\top} & \mathbf{c}_{6}^{\top} \\
* & * & * & \mathbf{c}_{7}^{\top} & \mathbf{c}_{8}^{\top} & \mathbf{c}_{9}^{\top} \\
\mathbf{c}_{1} & \mathbf{c}_{4} & \mathbf{c}_{7} & A_{1} & A_{2} & A_{3} \\
\mathbf{c}_{2} & \mathbf{c}_{5} & \mathbf{c}_{8} & A_{2} & A_{4} & A_{5} \\
\mathbf{c}_{3} & \mathbf{c}_{6} & \mathbf{c}_{9} & A_{3} & A_{5} & A_{6}
\end{array}\right),
$$

where stars are arbitrary numbers, $\mathbf{c}_{i}$ are vectors with two components, and $A_{i}$ are $2 \times 2$ matrices of the form (18). Let $\Phi$ be a finite measure on $\hat{\mathbb{R}}^{3} / \mathscr{O} \times Z_{2}^{c}$. Let $f^{0}(\mathbf{p})$ be a $\Phi$-equivalence class of measurable functions acting from $\left(\hat{\mathbb{R}}^{3} / \mathscr{O} \times Z_{2}^{c}\right)_{m}, 0 \leq m \leq 1$ to the set of nonnegative-definite symmetric matrices with unit trace such that $\mathbf{c}_{i}=\mathbf{0}$ and $A_{i}$ are proportional to the identity matrix. Let $f^{-}(\mathbf{p})$ be a $\Phi$-equivalence class of measurable functions acting from $\left(\hat{\mathbb{R}}^{3} / \mathscr{O} \times Z_{2}^{c}\right)_{2}$ to the set of nonnegative-definite symmetric matrices with unit trace such that $A_{i}$ are symmetric. Let $f^{+}(\mathbf{p})$ be a $\Phi$-equivalence class of measurable functions acting from $\left(\hat{\mathbb{R}}^{3} / \mathscr{O} \times Z_{2}^{c}\right)_{m}, 3 \leq m \leq 6$ to the set of nonnegative-definite symmetric matrices with unit trace. Consider matrices and functions of Table 6.

Let $f^{-i}(\mathbf{p})$ is obtained from $f^{-}(\mathbf{p})$ by replacing all $\mathbf{c}_{j}$ with $g_{i} \mathbf{c}_{i}$ and the vectors $(b, c)^{\top}$ in all $A_{j}$ with $g_{i}(b, c)^{\top}$. Let $f^{+i}(\mathbf{p})$ is obtained from $f^{+}(\mathbf{p})$ by replacing all $\mathbf{c}_{j}$ with $g_{i} \mathbf{c}_{i}$ and all $A_{j}$ with $g_{i} A_{j} g_{i}^{-1}$. 
Table 6 The matrices $g_{n}$ and the functions $j_{n}(\mathbf{p}, \mathbf{z})$ for the group $\mathscr{O} \times Z_{2}^{c}$

\begin{tabular}{lll}
\hline$n$ & $g_{n}$ & $j_{n}(\mathbf{p}, \mathbf{z})$ \\
\hline 1 & $\left(\begin{array}{ll}1 & 0 \\
0 & 1\end{array}\right)$ & $\cos \left(p_{3} z_{3}\right)\left[\cos \left(p_{1} z_{1}+p_{2} z_{2}\right)+\cos \left(p_{1} z_{2}+p_{2} z_{1}\right)\right]$ \\
2 & $\left(\begin{array}{rr}-1 & 0 \\
0 & 1\end{array}\right)$ & $\cos \left(p_{3} z_{3}\right)\left[\cos \left(-p_{1} z_{2}+p_{2} z_{1}\right)+\cos \left(p_{2} z_{2}-p_{1} z_{1}\right)\right]$ \\
3 & $\frac{1}{2}\left(\begin{array}{cc}1 & -\sqrt{3} \\
\sqrt{3} & 1\end{array}\right)$ & $2 \cos \left[\sqrt{2} p_{3}\left(z_{2}-z_{1}\right) / 2\right] \cos \left[\left(p_{2}-p_{1}\right)\left(z_{1}+z_{2}\right) / 2\right] \cos \left[\sqrt{2}\left(p_{1}+p_{2}\right) z_{3} / 2\right]$ \\
4 & $\frac{1}{2}\left(\begin{array}{cc}-1 & \sqrt{3} \\
\sqrt{3} & -1\end{array}\right)$ & $\cos \left[\sqrt{2}\left(p_{1}-p_{2}\right) z_{3} / 2\right]\left\{\cos \left[\left(-p_{1}-p_{2}+\sqrt{2} p_{3}\right) z_{1} / 2+\left(p_{1}+p_{2}+\sqrt{2} p_{3}\right) z_{2} / 2\right]\right.$ \\
& & $\left.+\cos \left[\left(-p_{1}-p_{2}-\sqrt{2} p_{3}\right) z_{1} / 2+\left(p_{1}+p_{2}-\sqrt{2} p_{3}\right) z_{2} / 2\right]\right\}$ \\
5 & $\frac{1}{2}\left(\begin{array}{cc}1 & \sqrt{3} \\
\sqrt{3} & -1\end{array}\right)$ & $2 \cos \left[\sqrt{2} p_{3}\left(z_{1}+z_{2}\right) / 2\right] \cos \left[\left(p_{2}-p_{1}\right)\left(z_{2}-z_{1}\right) / 2\right] \cos \left[\sqrt{2}\left(p_{1}+p_{2}\right) z_{3} / 2\right]$ \\
6 & $\frac{1}{2}\left(\begin{array}{cc}1 & -\sqrt{3} \\
-\sqrt{3}-1\end{array}\right)$ & $\cos \left[\sqrt{2}\left(p_{1}-p_{2}\right) z_{3} / 2\right]\left\{\cos \left[\left(p_{1}+p_{2}-\sqrt{2} p_{3}\right) z_{1} / 2+\left(p_{1}+p_{2}+\sqrt{2} p_{3}\right) z_{2} / 2\right]\right.$ \\
& & $\left.+\cos \left[\left(p_{1}+p_{2}+\sqrt{2} p_{3}\right) z_{1} / 2+\left(p_{1}+p_{2}-\sqrt{2} p_{3}\right) z_{2} / 2\right]\right\}$ \\
\hline
\end{tabular}

Theorem 9 (A cubic random field in the orthotropic class) The one-point correlation tensor of a homogeneous and $\left(\mathscr{O} \times Z_{2}^{c}, 3 A_{1 g} \oplus 3 E_{g}\right)$-isotropic random field $\mathrm{C}(\mathbf{x})$ is

$$
\langle\mathbf{C}(\mathbf{x})\rangle_{i j k l}=\sum_{m=1}^{3} C_{m} \mathscr{O} \times Z_{2}^{c} T_{i j k l}^{A_{1 g}, m, 1},
$$

where $C_{m} \in \mathbb{R}$. Its two-point correlation tensor has the form

$$
\begin{aligned}
\langle\mathrm{C}(\mathbf{x}), \mathrm{C}(\mathbf{y})\rangle= & \frac{1}{12}\left(\sum_{m=0}^{1} \int_{\left(\hat{\mathbb{R}}^{3} / \mathscr{O} \times Z_{2}^{c}\right)_{m}} \sum_{n=1}^{6} j_{n}(\mathbf{p}, \mathbf{y}-\mathbf{x}) f^{0}(\mathbf{p}) \mathrm{d} \Phi(\mathbf{p})\right. \\
& +\int_{\left(\hat{\mathbb{R}}^{3} / \mathscr{O} \times Z_{2}^{c}\right)_{2}} \sum_{n=1}^{6} j_{n}(\mathbf{p}, \mathbf{y}-\mathbf{x}) f^{-n}(\mathbf{p}) \mathrm{d} \Phi(\mathbf{p}) \\
& \left.+\sum_{m=3}^{6} \int_{\left(\hat{\mathbb{R}}^{3} / \mathscr{O} \times Z_{2}^{c}\right)_{m}} \sum_{n=1}^{6} j_{n}(\mathbf{p}, \mathbf{y}-\mathbf{x}) f^{+n}(\mathbf{p}) \mathrm{d} \Phi(\mathbf{p})\right) .
\end{aligned}
$$

The field has the form

$$
\begin{aligned}
\mathrm{C}(\mathbf{x})_{i j k l}= & \sum_{m=1}^{3} C_{m} \mathscr{O} \times Z_{2}^{c} T_{i j k l}^{A_{1 g}, m, 1}+\frac{1}{\sqrt{12}} \sum_{q=1}^{6} \sum_{n=1}^{48} \sum_{m=0}^{1} \int_{\left(\hat{\mathbb{R}}^{3} / \mathscr{O} \times Z_{2}^{c}\right)_{m}} u_{n}(\mathbf{p}, \mathbf{x}) \mathrm{d} Z_{q}^{0 n}(\mathbf{p})_{\mathscr{O} \times Z_{2}^{c}} T_{i j k l}^{q} \\
& +\frac{1}{\sqrt{12}} \sum_{q=1}^{6} \sum_{s=1}^{6} \sum_{n=8 s-7}^{8 s} \int_{\left(\hat{\mathbb{R}}^{3} / \mathscr{O} \times Z_{2}^{c}\right)_{2}} u_{n}(\mathbf{p}, \mathbf{x}) \mathrm{d} Z_{q}^{-n s}(\mathbf{p})_{\mathscr{O} \times Z_{2}^{c}} T_{i j k l}^{q} \\
& +\frac{1}{\sqrt{12}} \sum_{q=1}^{6} \sum_{s=1}^{6} \sum_{n=8 s-7}^{8 s} \sum_{m=3}^{6} \int_{\left(\hat{\mathbb{R}}^{3} / \mathscr{O} \times Z_{2}^{c}\right)_{m}} u_{n}(\mathbf{p}, \mathbf{x}) \mathrm{d} Z_{q}^{+n s}(\mathbf{p})_{\mathscr{O} \times Z_{2}^{c}} T_{i j k l}^{q},
\end{aligned}
$$

where $u_{n}(\mathbf{p}, \mathbf{x}), 1 \leq n \leq 8$ are different products of sines and cosines of angles in Table 6 , $\left(Z_{1}^{0 n}(\mathbf{p}), \ldots, Z_{9}^{0 n}(\mathbf{p})\right)^{\top}\left(\right.$ resp. $\left(Z_{1}^{-n s}(\mathbf{p}), \ldots, Z_{9}^{-n s}(\mathbf{p})\right)^{\top}$, resp. $\left.\left(Z_{1}^{+n s}(\mathbf{p}), \ldots, Z_{9}^{+n s}(\mathbf{p})\right)^{\top}\right)$ are centred uncorrelated $\mathrm{V}^{\mathscr{O}} \times Z_{2}^{c}$-valued random measures on $\left(\hat{\mathbb{R}}^{3} / \mathscr{O} \times Z_{2}^{c}\right)_{m}, 0 \leq m \leq 1$ (resp. 
on $\left(\hat{\mathbb{R}}^{3} / \mathscr{O} \times Z_{2}^{c}\right)_{2}$, resp. on $\left.\left(\hat{\mathbb{R}}^{3} / \mathscr{O} \times Z_{2}^{c}\right)_{2}, 3 \leq m \leq 6\right)$ with control measure $f^{0}(\mathbf{p}) \mathrm{d} \Phi(\mathbf{p})$ (resp. $f^{-s}(\mathbf{p}) \mathrm{d} \Phi(\mathbf{p})$, resp. $\left.f^{+s}(\mathbf{p}) \mathrm{d} \Phi(\mathbf{p})\right)$, and where

$$
\mathrm{T}_{i j k l}^{m}= \begin{cases}\mathscr{O} \times Z_{2}^{c} \mathbf{T}_{i j k l}^{A_{g}, m, 1}, & \text { if } 1 \leq m \leq 3 \\ \mathscr{O} \times Z_{2}^{c} \mathbf{T}_{i j k l}^{E^{2 g},\lfloor m / 2\rfloor-1, m \bmod 2+1}, & \text { if } 4 \leq m \leq 9 .\end{cases}
$$

\subsection{The Trigonal Class}

Introduce the following notation:

$$
\begin{aligned}
j_{10}(\mathbf{p}, \mathbf{z})= & \cos \left(p_{1} z_{1}+p_{3} z_{3}\right) \cos \left(p_{2} z_{2}\right) \\
& +\cos \left[\frac{1}{2}\left(p_{1}+\sqrt{3} p_{2}\right) z_{1}\right] \cos \left[\frac{1}{2}\left(\sqrt{3} p_{1}-p_{2}\right) z_{2}+p_{3} z_{3}\right] \\
& +\cos \left[\frac{1}{2}\left(p_{1}-\sqrt{3} p_{2}\right) z_{1}\right] \cos \left[\frac{1}{2}\left(-\sqrt{3} p_{1}+p_{2}\right) z_{2}+p_{3} z_{3}\right] .
\end{aligned}
$$

Theorem 10 (A trigonal random field in the trigonal class) The one-point correlation tensor of a homogeneous and $\left(D_{3} \times Z_{2}^{c}, 6 A_{1 g}\right)$-isotropic random field $\mathrm{C}(\mathbf{x})$ is

$$
\langle\mathrm{C}(\mathbf{x})\rangle_{i j k l}=\sum_{m=1}^{6} C_{m D_{3} \times Z_{2}^{c}} T_{i j k l}^{A_{1 g}, m, 1},
$$

where $C_{m} \in \mathbb{R}$. Its two-point correlation tensor has the form

$$
\langle\mathrm{C}(\mathbf{x}), \mathrm{C}(\mathbf{y})\rangle=\frac{1}{3} \int_{\hat{\mathbb{R}}^{3} / D_{3} \times Z_{2}^{c}} j_{10}(\mathbf{p}, \mathbf{y}-\mathbf{x}) f(\mathbf{p}) \mathrm{d} \Phi(\mathbf{p}),
$$

where $f(\mathbf{p})$ is the $\Phi$-equivalence class of measurable functions acting from $\hat{\mathbb{R}}^{3} / D_{3} \times Z_{2}^{c}$ to the set of nonnegative-definite symmetric linear operators on $\mathrm{V}_{3}^{D_{3} \times Z_{2}^{c}}$ with unit trace, and $\Phi$ is a finite measure on $\hat{\mathbb{R}}^{3} / D_{3} \times Z_{2}^{c}$. The field has the form

$$
\mathrm{C}(\mathbf{x})_{i j k l}=\sum_{m=1}^{6} C_{m D_{3} \times Z_{2}^{c}} T_{i j k l}^{A_{1 g}, m, 1}+\frac{1}{\sqrt{3}} \sum_{m=1}^{6} \sum_{n=1}^{12} \int_{\hat{\mathbb{R}}^{3} / D_{3} \times Z_{2}^{c}} u_{n}(\mathbf{p}, \mathbf{x}) \mathrm{d} Z^{m n}(\mathbf{p})_{D_{3} \times Z_{2}^{c}} T_{i j k l}^{A_{1 g}, m, 1},
$$

where $\left(Z^{1 n}(\mathbf{p}), \ldots, Z^{6 n}(\mathbf{p})\right)^{\top}$ are 12 centred uncorrelated $\mathrm{V}^{D_{3} \times Z_{2}^{c}}$-valued random measures on $\hat{\mathbb{R}}^{3} / D_{3} \times Z_{2}^{c}$ with control measure $f(\mathbf{p}) \mathrm{d} \Phi(\mathbf{p})$, and where $u_{n}(\mathbf{p}, \mathbf{x}), 1 \leq n \leq 4$ are four different products of sines and cosines of $p_{1} x_{1}+p_{3} x_{3}$ and $p_{2} x_{2}, u_{n}(\mathbf{p}, \mathbf{x}), 5 \leq n \leq 8$ are four different product of sines and cosines of $\frac{1}{2}\left(p_{1}+\sqrt{3} p_{2}\right) x_{1}$ and $\frac{1}{2}\left(\sqrt{3} p_{1}-p_{2}\right) x_{2}+p_{3} x_{3}$, $u_{n}(\mathbf{p}, \mathbf{x}), 9 \leq n \leq 12$ are four different product of sines and cosines of $\frac{1}{2}\left(p_{1}-\sqrt{3} p_{2}\right) x_{1}$ and $\frac{1}{2}\left(-\sqrt{3} p_{1}+p_{2}\right) x_{2}+p_{3} x_{3}$. 
Consider a $6 \times 6$ symmetric nonnegative-definite matrix with unit trace of the following structure

$$
\left(\begin{array}{cccccc}
* & * & * & * & * & c_{1} \\
* & * & * & * & * & c_{2} \\
* & * & * & * & * & c_{3} \\
* & * & * & * & * & c_{4} \\
* & * & * & * & * & c_{5} \\
c_{1} & c_{2} & c_{3} & c_{4} & c_{5} & *
\end{array}\right)
$$

where stars and $c_{i}$ are arbitrary numbers. Let $\Phi$ be a finite measure on $\hat{\mathbb{R}}^{3} / D_{6} \times Z_{2}^{c}$. Let $f^{0}(\mathbf{p})$ be a $\Phi$-equivalence class of measurable functions acting from $\left(\hat{\mathbb{R}}^{3} / D_{6} \times Z_{2}^{c}\right)_{m}, 0 \leq$ $m \leq 2$ to the set of nonnegative-definite symmetric matrices with unit trace such that $c_{i}=0$. Let $f^{+}(\mathbf{p})$ be a $\Phi$-equivalence class of measurable functions acting from $\left(\hat{\mathbb{R}}^{3} / D_{6} \times Z_{2}^{c}\right)_{m}$, $3 \leq m \leq 4$ to the set of nonnegative-definite symmetric matrices with unit trace, and let $f^{-}(\mathbf{p})$ be a $\Phi$-equivalence class of measurable functions acting from $\left(\hat{\mathbb{R}}^{3} / D_{6} \times Z_{2}^{c}\right)_{m}, 3 \leq$ $m \leq 4$ to the set of nonnegative-definite symmetric matrices with unit trace such that all $c_{i} \mathrm{~s}$ are multiplied by -1 .

Theorem 11 (A hexagonal random field in the trigonal class) The one-point correlation tensor of a homogeneous and $\left(D_{6} \times Z_{2}^{c}, 5 A_{1 g} \oplus B_{1 g}\right)$-isotropic random field $\mathrm{C}(\mathbf{x})$ is

$$
\langle\mathrm{C}(\mathbf{x})\rangle_{i j k l}=\sum_{m=1}^{5} C_{m D_{6} \times Z_{2}^{c}} T_{i j k l}^{A_{1 g}, 1,1},
$$

where $C_{m} \in \mathbb{R}$. Its two-point correlation tensor has the form

$$
\begin{aligned}
\langle\mathrm{C}(\mathbf{x}), \mathrm{C}(\mathbf{y})\rangle= & \frac{1}{6}\left(\sum_{m=0}^{2} \int_{\left(\hat{\mathbb{R}}^{3} / D_{6} \times Z_{2}^{c}\right)_{m}} \sum_{n=1}^{6} j_{n}(\mathbf{p}, \mathbf{y}-\mathbf{x}) f^{0}(\mathbf{p}) \mathrm{d} \Phi(\mathbf{p})\right. \\
& +\sum_{m=3}^{4} \int_{\left(\hat{\mathbb{R}}^{3} / D_{6} \times Z_{2}^{c}\right)_{m}} \sum_{n=1}^{3} j_{n}(\mathbf{p}, \mathbf{y}-\mathbf{x}) f^{+}(\mathbf{p}) \mathrm{d} \Phi(\mathbf{p}) \\
& \left.+\sum_{m=3}^{4} \int_{\left(\hat{\mathbb{R}}^{3} / D_{6} \times Z_{2}^{c}\right)_{m}} \sum_{n=4}^{6} j_{n}(\mathbf{p}, \mathbf{y}-\mathbf{x}) f^{-}(\mathbf{p}) \mathrm{d} \Phi(\mathbf{p})\right) .
\end{aligned}
$$

The field has the form

$$
\begin{aligned}
\mathrm{C}(\mathbf{x})_{i j k l}= & \sum_{m=1}^{5} C_{m} D_{6} \times Z_{2}^{c} \top_{i j k l}^{A_{1 g}, m, 1}+\frac{1}{\sqrt{6}} \sum_{q=1}^{6} \sum_{n=1}^{24} \sum_{m=0}^{2} \int_{\left(\hat{\mathbb{R}}^{3} / D_{6} \times Z_{2}^{c}\right)_{m}} u_{n}(\mathbf{p}, \mathbf{x}) \mathrm{d} Z_{q}^{0 n}(\mathbf{p})_{D_{6} \times Z_{2}^{c}} T_{i j k l}^{q} \\
& +\frac{1}{\sqrt{6}} \sum_{q=1}^{6} \sum_{s=1}^{6} \sum_{n=4 s-3}^{4 s} \sum_{m=3}^{4} \int_{\left(\hat{\mathbb{R}}^{3} / D_{6} \times Z_{2}^{c}\right)_{m}} u_{n}(\mathbf{p}, \mathbf{x}) \mathrm{d} Z_{q}^{+n s}(\mathbf{p})_{D_{6} \times Z_{2}^{c}} T_{i j k l}^{q} \\
& +\frac{1}{\sqrt{6}} \sum_{q=1}^{9} \sum_{s=1}^{6} \sum_{n=4 s-3}^{4 s} \sum_{m=3}^{4} \int_{\left(\hat{\mathbb{R}}^{3} / D_{6} \times Z_{2}^{c}\right)_{m}} u_{n}(\mathbf{p}, \mathbf{x}) \mathrm{d} Z_{q}^{-n s}(\mathbf{p})_{D_{6} \times Z_{2}^{c}} T_{i j k l}^{q},
\end{aligned}
$$

where $\left(Z_{1}^{0 n}(\mathbf{p}), \ldots, Z_{6}^{0 n}(\mathbf{p})\right)^{\top} \quad\left(\operatorname{resp} . \quad\left(Z_{1}^{+n s}(\mathbf{p}), \ldots, Z_{6}^{+n s}(\mathbf{p})\right)^{\top}, \quad\right.$ resp. $\quad\left(Z_{1}^{-n s}(\mathbf{p}), \ldots\right.$, $\left.\left.Z_{6}^{-n s}(\mathbf{p})\right)^{\top}\right)$ are centred uncorrelated $\mathrm{V}^{D_{6} \times Z_{2}^{c}}$-valued random measures on $\left(\hat{\mathbb{R}}^{3} / D_{6} \times Z_{2}^{c}\right)_{m}$, 
$0 \leq m \leq 2$ (resp. on $\left.\left(\hat{\mathbb{R}}^{3} / D_{6} \times Z_{2}^{c}\right)_{m}, 3 \leq m \leq 4\right)$ with control measure $f^{0}(\mathbf{p}) \mathrm{d} \Phi(\mathbf{p})$ (resp. $f^{+}(\mathbf{p}) \mathrm{d} \Phi(\mathbf{p})$, resp. $\left.f^{-}(\mathbf{p}) \mathrm{d} \Phi(\mathbf{p})\right), u_{n}(\mathbf{p}, \mathbf{x}), 1 \leq n \leq 8$ are different product of sines and cosines of angles in Table 5, and where

$$
T_{i j k l}^{q}= \begin{cases}D_{6} \times Z_{2}^{c} \mathrm{~T}_{i j k l}^{A_{1 g}, q, 1}, & \text { if } 1 \leq q \leq 5, \\ D_{6} \times Z_{2}^{c} \mathrm{~T}_{i j k l}^{B_{1 g}, m, 1}, & \text { otherwise. }\end{cases}
$$

\subsection{The Tetragonal Class}

Theorem 12 (A tetragonal random field in the tetragonal class) The one-point correlation tensor of a homogeneous and $\left(D_{4} \times Z_{2}^{c}, 6 A_{1 g}\right)$-isotropic random field $\mathrm{C}(\mathbf{x})$ is

$$
\langle\mathbf{C}(\mathbf{x})\rangle_{i j k l}=\sum_{m=1}^{6} C_{m D_{4} \times Z_{2}^{c}} \mathrm{~T}_{i j k l}^{A_{1 g}, m, 1}
$$

where $C_{m} \in \mathbb{R}$. Its two-point correlation tensor has the form

$$
\begin{aligned}
\langle\mathbf{C}(\mathbf{x}), \mathbf{C}(\mathbf{y})\rangle= & \frac{1}{2} \int_{\hat{\mathbb{R}}^{3} / D_{4} \times Z_{2}^{c}}\left[\cos \left(p_{1}\left(x_{1}-y_{1}\right)\right) \cos m\left(p_{2}\left(x_{2}-y_{2}\right)\right)\right. \\
& \left.+\cos \left(p_{1}\left(x_{2}-y_{2}\right)\right) \cos \left(p_{2}\left(x_{1}-y_{1}\right)\right)\right] \cos \left(p_{3}\left(x_{3}-y_{3}\right)\right) f(\mathbf{p}) \mathrm{d} \Phi(\mathbf{p}),
\end{aligned}
$$

where $f(\mathbf{p})$ is a $\Phi$-equivalence class of measurable functions acting from $\hat{\mathbb{R}}^{3} / D_{4} \times Z_{2}^{c}$ to the set of nonnegative-definite symmetric linear operators on $\mathrm{V}^{D_{4} \times Z_{2}^{c}}$ with unit trace, and $\Phi$ is a finite measure on $\hat{\mathbb{R}}^{3} / D_{4} \times Z_{2}^{c}$. The field has the form

$$
\mathrm{C}(\mathbf{x})_{i j k l}=\sum_{m=1}^{6} C_{m D_{4} \times Z_{2}^{c}} \top_{i j k l}^{A_{1 g}, m, 1}+\frac{1}{\sqrt{2}} \sum_{m=1}^{6} \sum_{n=1}^{16} \int_{\hat{\mathbb{R}}^{3} / D_{4} \times Z_{2}^{c}} u_{n}(\mathbf{p}, \mathbf{x}) \mathrm{d} Z^{m n}(\mathbf{p})_{D_{4} \times Z_{2}^{c}} \top_{i j k l}^{A_{1 g}, m, 1},
$$

where $\left(Z^{1 n}(\mathbf{p}), \ldots, Z^{6 n}(\mathbf{p})\right)^{\top}$ are 16 centred uncorrelated $\mathrm{V}^{D_{4} \times Z_{2}^{c}}$-valued random measures on $\hat{\mathbb{R}}^{3} / D_{4} \times Z_{2}^{c}$ with control measure $f(\mathbf{p}) \mathrm{d} \Phi(\mathbf{p})$, and where $u_{n}(\mathbf{p}, \mathbf{x})$ are eight different product of sines and cosines of $p_{r} x_{r}$ for $1 \leq n \leq 8$ and eight different product of sines and cosines of $p_{1} x_{2}, p_{2} x_{1}$, and $p_{3} x_{3}$ for $9 \leq n \leq 16$.

Consider a $6 \times 6$ symmetric nonnegative-definite matrix with unit trace of the structure (19). Let $\Phi$ be a finite measure on $\hat{\mathbb{R}}^{3} / D_{8} \times Z_{2}^{c}$. Let $f^{0}(\mathbf{p})$ be a $\Phi$-equivalence class of measurable functions acting from $\left(\hat{\mathbb{R}}^{3} / D_{8} \times Z_{2}^{c}\right)_{m}, 0 \leq m \leq 1$ to the set of nonnegative-definite symmetric matrices with unit trace such that $c_{i}=0$. Let $f^{+}(\mathbf{p})$ be a $\Phi$-equivalence class of measurable functions acting from $\left(\hat{\mathbb{R}}^{3} / D_{8} \times Z_{2}^{c}\right)_{m}, 2 \leq m \leq 4$ to the set of nonnegativedefinite symmetric matrices with unit trace, and let $f^{-}(\mathbf{p})$ be a $\Phi$-equivalence class of measurable functions acting from $\left(\hat{\mathbb{R}}^{3} / D_{8} \times Z_{2}^{c}\right)_{m}, 2 \leq m \leq 4$ to the set of nonnegative-definite symmetric matrices with unit trace such that all $c_{i}$ s are multiplied by -1 . 
Introduce the following notation.

$$
\begin{aligned}
j_{13}^{+}(\mathbf{p}, \mathbf{z})= & 2 \cos \left(p_{3} z_{3}\right)\left[\cos \left(p_{1} z_{1}+p_{2} z_{2}\right)+\cos \left(p_{2} z_{1}-p_{1} z_{2}\right)\right. \\
& +\cos \left(\left(p_{1}+p_{2}\right)\left(z_{1}+z_{2}\right) / \sqrt{2}\right) \\
& \left.+\cos \left(\left(p_{2} z_{2}-p_{1} z_{1}\right) / \sqrt{2}-p_{3} z_{3}\right) \cos \left(\left(p_{1} z_{2}+p_{2} z_{1}\right) / \sqrt{2}\right)\right], \\
j_{13}^{-}(\mathbf{p}, \mathbf{z})= & \cos \left(p_{3} z_{3}\right)\left[2 \cos \left(p_{1} z_{1}-p_{2} z_{2}\right)+2 \cos \left(p_{2} z_{1}+p_{1} z_{2}\right)\right. \\
& \left.+\cos \left(\left(p_{1} z_{1}+p_{2} z_{2}\right) / \sqrt{2}\right) \cos \left(\left(p_{2} z_{1}-p_{1} z_{2}\right) / \sqrt{2}\right)\right] .
\end{aligned}
$$

Theorem 13 (An octagonal random field in the tetragonal class) The one-point correlation tensor of a homogeneous and $\left(D_{8} \times Z_{2}^{c}, 5 A_{1 g} \oplus B_{1 g}\right)$-isotropic random field $\mathrm{C}(\mathbf{x})$ is

$$
\langle\mathrm{C}(\mathbf{x})\rangle_{i j k l}=\sum_{m=1}^{5} C_{m D_{8} \times Z_{2}^{c}} T_{i j k l}^{A_{1 g}, 1,1},
$$

where $C_{m} \in \mathbb{R}$. Its two-point correlation tensor has the form

$$
\begin{aligned}
\langle\mathrm{C}(\mathbf{x}), \mathrm{C}(\mathbf{y})\rangle= & \frac{1}{4}\left(\sum_{m=0}^{1} \int_{\left(\hat{\mathbb{R}}^{3} / D_{8} \times Z_{2}^{c}\right)_{m}}\left(j_{13}^{+}(\mathbf{p}, \mathbf{y}-\mathbf{x})+j_{13}^{-}(\mathbf{p}, \mathbf{y}-\mathbf{x})\right) f^{0}(\mathbf{p}) \mathrm{d} \Phi(\mathbf{p})\right. \\
& +\sum_{m=2}^{4} \int_{\left(\hat{\mathbb{R}}^{3} / D_{8} \times Z_{2}^{c}\right)_{m}} j_{13}^{+}(\mathbf{p}, \mathbf{y}-\mathbf{x}) f^{+}(\mathbf{p}) \mathrm{d} \Phi(\mathbf{p}) \\
& \left.+\sum_{m=2}^{4} \int_{\left(\hat{\mathbb{R}}^{3} / D_{8} \times Z_{2}^{c}\right)_{m}} j_{13}^{-}(\mathbf{p}, \mathbf{y}-\mathbf{x}) f^{-}(\mathbf{p}) \mathrm{d} \Phi(\mathbf{p})\right) .
\end{aligned}
$$

The field has the form

$$
\begin{aligned}
\mathrm{C}(\mathbf{x})_{i j k l}= & \sum_{m=1}^{5} C_{m} D_{8} \times Z_{2}^{c} \mathrm{~T}_{i j k l}^{A_{1 g}, m, 1}+\frac{1}{2} \sum_{q=1}^{6} \sum_{n=1}^{32} \sum_{m=0}^{1} \int_{\left(\hat{\mathbb{R}}^{3} / D_{8} \times Z_{2}^{c}\right)_{m}} u_{n}(\mathbf{p}, \mathbf{x}) \mathrm{d} Z_{q}^{0 n}(\mathbf{p})_{D_{8} \times Z_{2}^{c}} T_{i j k l}^{q} \\
& +\frac{1}{2} \sum_{q=1}^{6} \sum_{n=1}^{16} \sum_{m=2}^{4} \int_{\left(\hat{\mathbb{R}}^{3} / D_{8} \times Z_{2}^{c}\right)_{m}} u_{n}(\mathbf{p}, \mathbf{x}) \mathrm{d} Z_{q}^{+n}(\mathbf{p})_{D_{8} \times Z_{2}^{c}} T_{i j k l}^{q} \\
& +\frac{1}{2} \sum_{q=1}^{6} \sum_{n=17}^{32} \sum_{m=2}^{4} \int_{\left(\hat{\mathbb{R}}^{3} / D_{8} \times Z_{2}^{c}\right)_{m}} u_{n}(\mathbf{p}, \mathbf{x}) \mathrm{d} Z_{q}^{-n}(\mathbf{p})_{D_{8} \times Z_{2}^{c}} T_{i j k l}^{q},
\end{aligned}
$$

where $\left(Z_{1}^{0 n}(\mathbf{p}), \ldots, Z_{6}^{0 n}(\mathbf{p})\right)^{\top}\left(\operatorname{resp} .\left(Z_{1}^{+n}(\mathbf{p}), \ldots, Z_{6}^{+n}(\mathbf{p})\right)^{\top}\right.$, resp. $\left.\left(Z_{1}^{-n}(\mathbf{p}), \ldots, Z_{6}^{-n}(\mathbf{p})\right)^{\top}\right)$ are centred uncorrelated $\mathrm{V}^{D_{8} \times Z_{2}^{c}}$-valued random measures on $\left(\hat{\mathbb{R}}^{3} / D_{8} \times Z_{2}^{c}\right)_{m}, 0 \leq$ $m \leq 1$ (resp. on $\left.\left(\hat{\mathbb{R}}^{3} / D_{8} \times Z_{2}^{c}\right)_{m}, 2 \leq m \leq 4\right)$ with control measure $f^{0}(\mathbf{p}) \mathrm{d} \Phi(\mathbf{p})$ (resp. $f^{+}(\mathbf{p}) \mathrm{d} \Phi(\mathbf{p})$, resp. $\left.f^{-}(\mathbf{p}) \mathrm{d} \Phi(\mathbf{p})\right), u_{n}(\mathbf{p}, \mathbf{x}), 1 \leq n \leq 8$ are different product of sines and cosines of angles in Table 5, and where

$$
T_{i j k l}^{q}= \begin{cases}D_{8} \times Z_{2}^{c} \top_{i j k l}^{A_{1 g}, q, 1}, & \text { if } 1 \leq q \leq 5, \\ D_{8} \times Z_{2}^{c} \top_{i j k l}^{B_{1 g}, m, 1}, & \text { otherwise. }\end{cases}
$$




\subsection{The Transverse Isotropic Class}

Theorem 14 (A transverse isotropic random field in the transverse isotropic class) The onepoint correlation tensor of the homogeneous and $\left(\mathrm{O}(2) \times Z_{2}^{c}, 5 U^{0 g g}\right)$-isotropic mean-square continuous random field $\mathrm{C}(\mathbf{x})$ has the form

$$
\langle\mathrm{C}(\mathbf{x})\rangle=\sum_{m=1}^{5} C_{m \mathrm{O}(2) \times Z_{2}^{c}} T_{i j k l}^{U^{0 g g}, m, 1},
$$

where $C_{m} \in \mathbb{R}$. Its two-point correlation tensor has the form

$$
\begin{aligned}
\langle\mathbf{C}(\mathbf{x}), \mathbf{C}(\mathbf{y})\rangle= & \int_{\hat{\mathbb{R}}^{3} / \mathrm{O}(2) \times Z_{2}^{c}} J_{0}\left(\sqrt{\left(p_{1}^{2}+p_{2}^{2}\right)\left(\left(y_{1}-x_{1}\right)^{2}+\left(y_{2}-x_{2}\right)^{2}\right)}\right) \\
& \times \cos \left(p_{3}\left(y_{3}-x_{3}\right)\right) f(\mathbf{p}) \mathrm{d} \Phi(\mathbf{p}),
\end{aligned}
$$

where $\Phi$ is a measure on $\hat{\mathbb{R}}^{3} / \mathrm{O}(2) \times Z_{2}^{c}$, and $f(\mathbf{p})$ is a $\Phi$-equivalence class of measurable functions on $\hat{\mathbb{R}}^{3} / \mathrm{O}(2) \times Z_{2}^{c}$ with values in the compact set of all nonnegative-definite linear operators in the space $\mathrm{V}^{\mathrm{O}(2)} \times Z_{2}^{c}$ with unit trace. The field has the form

$$
\begin{aligned}
\mathrm{C}(\mathbf{x})= & \sum_{m=1}^{5} C_{m} \mathrm{O}(2) \times Z_{2}^{c} \\
& T_{i j k l}^{0 \otimes A, m, 1} \\
& +\sum_{m=1}^{5} \int_{\hat{\mathbb{R}}^{3} / \mathrm{O}(2) \times Z_{2}^{c}} J_{0}\left(\sqrt{\left(p_{1}^{2}+p_{2}^{2}\right)\left(x_{1}^{2}+x_{2}^{2}\right)}\right) \\
& \times\left(\cos \left(p_{3} x_{3}\right) \mathrm{d} Z^{01 m}(\mathbf{p})_{\mathrm{O}(2) \times Z_{2}^{c}} T_{i j k l}^{U^{0 g g}, m, 1}+\sin \left(p_{3} x_{3}\right) \mathrm{d} Z^{02 m}(\mathbf{p})_{\mathrm{O}(2) \times Z_{2}^{c}} T_{i j k l}^{U^{0 g g}, m, 1}\right) \\
& +\sqrt{2} \sum_{\ell=1}^{\infty} \sum_{m=1}^{5} \int_{\hat{\mathbb{R}}^{3} / \mathrm{O}(2) \times Z_{2}^{c}} J_{\ell}\left(\sqrt{\left(p_{1}^{2}+p_{2}^{2}\right)\left(x_{1}^{2}+x_{2}^{2}\right)}\right) \\
& \times\left(\cos \left(p_{3} x_{3}\right) \cos \left(\ell \varphi_{p}\right) \mathrm{d} Z^{\ell 1 m}(\mathbf{p})_{\mathrm{O}(2) \times Z_{2}^{c}} T_{i j k l}^{U^{0 g g}, m, 1}\right. \\
& +\cos \left(p_{3} x_{3}\right) \sin \left(\ell \varphi_{p}\right) \mathrm{d} Z^{\ell 2 m}(\mathbf{p})_{\mathrm{O}(2) \times Z_{2}^{c}} T_{i j k l}^{U^{0 g g}, m, 1} \\
& +\sin \left(p_{3} x_{3}\right) \cos \left(\ell \varphi_{p}\right) \mathrm{d} Z^{\ell 3 m}(\mathbf{p})_{\mathrm{O}(2) \times Z_{2}^{c}} T_{i j k l}^{U^{0 g g}, m, 1} \\
& \left.+\sin \left(p_{3} x_{3}\right) \sin \left(\ell \varphi_{p}\right) \mathrm{d} Z^{\ell 4 m}(\mathbf{p})_{\mathrm{O}(2) \times Z_{2}^{c}} T_{i j k l}^{U^{0 g g}, m, 1}\right)
\end{aligned}
$$

where $\left(Z^{\ell i 1}(\mathbf{p}), \ldots, Z^{\ell i 5}(\mathbf{p})\right)^{\top}$ are centred uncorrelated $\mathrm{V}^{\mathrm{O}(2) \times Z_{2}^{c}}$-valued random measures on $\hat{\mathbb{R}}^{3} / \mathrm{O}(2) \times Z_{2}^{c}$ with control measure $f(\mathbf{p}) \mathrm{d} \Phi(\mathbf{p})$.

\subsection{The Cubic Class}

Theorem 15 (A cubic random field in the cubic class) The one-point correlation tensor of the homogeneous and $\left(\mathscr{O} \times Z_{2}^{c}, 3 A_{1 g}\right)$-isotropic mean-square continuous random field $\mathrm{C}(\mathbf{x})$ has the form

$$
\langle\mathrm{C}(\mathbf{x})\rangle=\sum_{m=1}^{3} C_{m} \mathscr{O} \times Z_{2}^{c} \mathrm{~T}_{i j k l}^{A_{1 g}, m, 1},
$$


where $C_{m} \in \mathbb{R}$. Its two-point correlation tensor has the form

$$
\langle\mathrm{C}(\mathbf{x}), \mathrm{C}(\mathbf{y})\rangle=\int_{\hat{\mathbb{R}}^{3} / \mathscr{O} \times Z_{2}^{c}} \sum_{m=0}^{8} j_{m}(\mathbf{x}-\mathbf{y}, \mathbf{p}) f(\mathbf{p}) \mathrm{d} \Phi(\mathbf{p}),
$$

where the functions $j_{m}(\mathbf{z}, \mathbf{p})$ are shown in Table $6, \Phi$ is a measure on $\hat{\mathbb{R}}^{3} / \mathscr{O} \times Z_{2}^{c}$, and $f(\mathbf{p})$ is a $\Phi$-equivalence class of measurable functions on $\hat{\mathbb{R}}^{3} / \mathscr{O} \times Z_{2}^{c}$ with values in the compact set of all nonnegative-definite linear operators in the space $\mathrm{V}^{\mathscr{O}} \times Z_{2}^{c}$ with unit trace. The field has the form

$$
\mathrm{C}(\mathbf{x})=\sum_{m=1}^{3} C_{m} \mathscr{O} \times Z_{2}^{c} \mathbf{T}_{i j k l}^{A_{1 g}, m, 1}+\sum_{m=1}^{3} \sum_{n=1}^{48} \int_{\hat{\mathbb{R}}^{3} / \mathscr{O} \times Z_{2}^{c}} u_{n}(\mathbf{x}, \mathbf{p}) \mathrm{d} Z^{m n}(\mathbf{p})_{\mathscr{O} \times Z_{2}^{c}} \mathbf{T}_{i j k l}^{A_{1 g}, m, 1},
$$

where $\left(Z^{1 n}(\mathbf{p}), \ldots, Z^{3 n}(\mathbf{p})\right)^{\top}$ are 48 centred uncorrelated $\mathrm{V}^{\mathscr{O}} \times Z_{2}^{c}$-valued random measures on $\hat{\mathbb{R}}^{3} / \mathscr{O} \times Z_{2}^{c}$ with control measure $f(\mathbf{p}) \mathrm{d} \mu(\mathbf{p})$, and where $u_{n}(\mathbf{x}, \mathbf{p})$ are different products of sines and cosines of angles from Table 6.

\subsection{The Isotropic Class}

Theorem 16 (An isotropic random field in the isotropic class) The one-point correlation tensor of the homogeneous and $\left(\mathrm{O}(3), 2 U^{0 g}\right)$-isotropic mean-square continuous random field $\mathrm{C}(\mathbf{x})$ has the form

$$
\langle\mathrm{C}(\mathbf{x})\rangle=C_{1} \delta_{i j} \delta_{k l}+C_{2}\left(\delta_{i k} \delta_{j l}+\delta_{i l} \delta_{j k}\right), \quad C_{m} \in \mathbb{R} .
$$

Its two-point correlation tensor has the form

$$
\langle\mathrm{C}(\mathbf{x}), \mathrm{C}(\mathbf{y})\rangle=\int_{0}^{\infty} \frac{\sin (\lambda\|\mathbf{y}-\mathbf{x}\|)}{\lambda\|\mathbf{y}-\mathbf{x}\|} f(\lambda) \mathrm{d} \Phi(\lambda),
$$

where $\Phi(\lambda)$ is a finite measure on $[0, \infty)$,

$$
f(\lambda)=\left(\begin{array}{cc}
v_{1}(\lambda) & v_{2}(\lambda) \\
v_{2}(\lambda) & 1-v_{1}(\lambda)
\end{array}\right)
$$

and where $\mathbf{v}(\lambda)=\left(v_{1}(\lambda), v_{2}(\lambda)\right)^{\top}$ is a $\Phi$-equivalence class of measurable functions on $[0, \infty)$ taking values in the closed disk $\left(v_{1}(\lambda)-1 / 2\right)^{2}+v_{2}^{2}(\lambda) \leq 1 / 4$. The field itself has the form

$$
\begin{aligned}
\mathrm{C}_{i j k l}(\rho, \theta, \varphi)= & C_{1} \delta_{i j} \delta_{k l}+C_{2}\left(\delta_{i k} \delta_{j l}+\delta_{i l} \delta_{j k}\right)+2 \sqrt{\pi} \sum_{\ell=0}^{\infty} \sum_{m=\ell}^{\ell} S_{\ell}^{m}(\theta, \varphi) \int_{0}^{\infty} j_{\ell}(\lambda \rho) \\
& \times\left(\mathrm{o}(3) \mathrm{T}_{i j k l}^{0,1,0} \mathrm{~d} Z_{\ell 1}^{m}(\lambda)+\mathrm{o}(3) \mathrm{T}_{i j k l}^{0,2,0} \mathrm{~d} Z_{\ell 2}^{m}(\lambda)\right),
\end{aligned}
$$

where $\left(Z_{\ell 1}^{m}, Z_{\ell 2}^{m}\right)^{\top}$ is the set of mutually uncorrelated $\mathrm{V}^{\mathrm{O}(3)}$-valued random measures with $f(\lambda) \mathrm{d} \Phi(\lambda)$ as their common control measure. 


\section{Conclusions}

Hooke's law describes the physical phenomenon of elasticity and belongs to the family of linear constitutive laws, see [25]. In general, a linear constitutive law is an element of a subspace of the tensor product $V^{\otimes(p+q)}$, where $p$ (resp. $q$ ) is the rank of tensors in the first (resp. second) state tensor space. Denote by $U$ the restriction of the representation $g \mapsto$ $g^{\otimes(p+q)}$ to the above subspace. Consider $U$ as a group action. The orbit types of this action are called the classes of the phenomenon under consideration (e.g., photoelasticity classes, piezoelectricity classes and so on). All symmetry classes of all possible linear constitutive laws were described in [25, 26].

For each class, one can consider its fixed point set $\mathrm{V}^{H} \subset V^{\otimes(p+q)}$, a group $K$ with $H \subseteq K \subseteq N(H)$, and the restriction $U$ of the representation $g \mapsto g^{\otimes(p+q)}$ of the group $K$ to $\mathrm{V}^{H}$. Calculating the general form of the one-point and two-point correlation tensors of the corresponding homogeneous and $(K, U)$-isotropic random field and the spectral expansion of the field in terms of stochastic integrals with respect to orthogonal scattered random measures is an interesting research question.

There are two principal uses of the results obtained here. The first one is to model and simulate any statistically wide-sense homogeneous and isotropic, linear hyperelastic, random medium. One example is a polycrystal made of grains belonging to a specific crystal class, while another example is a mesoscale continuum defined through upscaling of a random material on scales smaller than the RVE; if the upscaling is conducted on the RVE level, there is no spatial randomness and the continuum model is deterministic. Here one would proceed in the following steps:

- for a given microstructure, determine the one- and two-point statistics using some experimental and/or image-based computational methods;

- calibrate the entire correlation structure of the elasticity TRF;

- simulate the realisations of this TRF.

The second application of our results is their use as input of a random mesoscale continuum (Fig. 1(c)) into stochastic field equations such as SPDEs and SFEs.

Acknowledgements We are grateful to the two anonymous referees for their remarks that helped to improve the paper significantly.

Open Access This article is distributed under the terms of the Creative Commons Attribution 4.0 International License (http://creativecommons.org/licenses/by/4.0/), which permits unrestricted use, distribution, and reproduction in any medium, provided you give appropriate credit to the original author(s) and the source, provide a link to the Creative Commons license, and indicate if changes were made.

\section{References}

1. Altmann, S.L., Herzig, P.: Point-Group Theory Tables. Oxford Science Publications Clarendon, Oxford (1994)

2. Auffray, N., Kolev, B., Petitot, M.: On anisotropic polynomial relations for the elasticity tensor. J. Elast. 115(1), 77-103 (2014)

3. Bourbaki, N.: Integration. II. Chapters 7-9. In: Elements of Mathematics (Berlin). Springer, Berlin (2004)

4. Brock, C. (ed.): International Tables for Crystallography, vol. A-G, 4th edn. Wiley, New York (2014)

5. Cramér, H.: On the theory of stationary random processes. Ann. Math. (2) 41, 215-230 (1940)

6. Duistermaat, J.J., Kolk, J.A.C.: Lie Groups. Universitext. Springer, Berlin (2000)

7. Forte, S., Vianello, M.: Symmetry classes for elasticity tensors. J. Elast. 43(2), 81-108 (1996) 
8. Godunov, S.K., Gordienko, V.M.: Clebsch-Gordan coefficients in the case of various choices of bases of unitary and orthogonal representations of the groups SU(2) and SO(3). Sib. Mat. Zh. 45(3), 540-557 (2004)

9. Golubitsky, M., Stewart, I., Schaeffer, D.G.: Singularities and Groups in Bifurcation Theory. Vol. II. Applied Mathematical Sciences, vol. 69. Springer, New York (1988)

10. Gordienko, V.M.: Matrix elements of real representations of the groups $\mathrm{O}(3)$ and $\mathrm{SO}(3)$. Sib. Mat. Zh. 43(1), 51-63 (2002)

11. Guilleminot, J., Soize, C.: Non-Gaussian positive-definite matrix-valued random fields with constrained eigenvalues: application to random elasticity tensors with uncertain material symmetries. Int. J. Numer. Methods Eng. 88(11), 1128-1151 (2011)

12. Guilleminot, J., Soize, C.: Generalized stochastic approach for constitutive equation in linear elasticity: a random matrix model. Int. J. Numer. Methods Eng. 90(5), 613-635 (2012)

13. Guilleminot, J., Soize, C.: On the statistical dependence for the components of random elasticity tensors exhibiting material symmetry properties. J. Elast. 111(2), 109-130 (2013)

14. Guilleminot, J., Soize, C.: Stochastic model and generator for random fields with symmetry properties: application to the mesoscopic modeling of elastic random media. Multiscale Model. Simul. 11(3), 840870 (2013)

15. Guilleminot, J., Soize, C.: Itô SDE-based generator for a class of non-Gaussian vector-valued random fields in uncertainty quantification. SIAM J. Sci. Comput. 36(6), A2763-A2786 (2014)

16. Hermann, C.: Zur systematischen Strukturtheorie I. Eine neue Raumgruppensymbolik. Z. Kristallogr. 68, 257-287 (1928)

17. Hofmann, K.H., Morris, S.A.: The Structure of Compact Groups. A Primer for the Student-a Handbook for the Expert, 3rd edn. De Gruyter Studies in Mathematics, vol. 25. De Gruyter, Berlin (2013)

18. Karhunen, K.: Über lineare Methoden in der Wahrscheinlichkeitsrechnung. Ann. Acad. Sci. Fenn., a 1. Math.-Phys. 1947(37), 79 (1947)

19. Lord, G.J., Powell, C.E., Shardlow, T.: An Introduction to Computational Stochastic PDEs. Cambridge Texts in Applied Mathematics. Cambridge University Press, New York (2014)

20. Malyarenko, A., Ostoja-Starzewski, M.: The spectral expansion of the elasticity random field. In: Sivasundaram, S. (ed.) 10th International Conference on Mathematical Problems in Engineering, Aerospace and Sciences: ICNPAA2014. AIP Conf. Proc., vol. 1637, pp. 647-655 (2014)

21. Malyarenko, A., Ostoja-Starzewski, M.: A random field formulation of Hooke's law in all elasticity classes (2016). arXiv:1602.09066v2 [math-ph]

22. Malyarenko, A., Ostoja-Starzewski, M.: Spectral expansions of homogeneous and isotropic tensorvalued random fields. Z. Angew. Math. Phys. 67(3), 59 (2016)

23. Mauguin, C.: Sur le symbolisme des groupes de répetition ou de symétrie des assemblages cristallins. Z. Kristallogr. 76, 542-558 (1931)

24. Mulliken, R.S.: Electronic structures of polyatomic molecules and valence. IV. Electronic states, quantum theory of the double bond. Phys. Rev. 43, 279-302 (1933)

25. Olive, M., Auffray, N.: Symmetry classes for even-order tensors. Math. Mech. Compl. Syst. 1(2), 177210 (2013)

26. Olive, M., Auffray, N.: Symmetry classes for odd-order tensors. Z. Angew. Math. Mech. 94(5), 421-447 (2014)

27. Ostoja-Starzewski, M.: Microstructural Randomness and Scaling in Mechanics of Materials. CRC Series: Modern Mechanics and Mathematics. Chapman \& Hall/CRC, Boca Raton (2008)

28. Ostoja-Starzewski, M.: Stochastic finite elements: where is the physics? Theor. Appl. Mech. 38(4), 379396 (2011)

29. Ostoja-Starzewski, M., Kale, S., Karimi, P., Malyarenko, A., Raghavan, B., Ranganathan, S., Zhang, J.: Chapter Two-Scaling to RVE in random media. In: Bordas, S.P., Balint, D.S. (eds.) Advances in Applied Mechanics, vol. 49, pp. 111-211. Elsevier, Amsterdam (2016)

30. Ranganathan, S.I., Ostoja-Starzewski, M.: Towards scaling laws in random polycrystals. Int. J. Eng. Sci. 47(11-12), 1322-1330 (2009)

31. Schoenberg, I.J.: Metric spaces and completely monotone functions. Ann. Math. (2) 39(4), 811-841 (1938)

32. Schönfließ, A.: Krystallsysteme und Krystallstructur. Teubner, Leipzig (1891)

33. Sena, M.P., Ostoja-Starzewski, M., Costa, L.: Stiffness tensor random fields through upscaling of planar random materials. Probab. Eng. Mech. 34, 131-156 (2013)

34. Tonti, E.: The Mathematical Structure of Classical and Relativistic Physics. a General Classification Diagram. Modeling and Simulation in Science. Engineering and Technology. Springer, New York (2013)

35. Truesdell, C.A. III: A First Course in Rational Continuum Mechanics. Vol. 1. General Concepts, 2nd edn. Pure and Applied Mathematics, vol. 71. Academic Press, Inc., Boston (1991) 
36. Yaglom, A.M.: Homogeneous and isotropic turbulence in a viscous compressible fluid. Izv. Akad. Nauk SSSR, Ser. Geogr. Geofiz. 12, 501-522 (1948)

37. Yaglom, A.M.: Second-order homogeneous random fields. In: Proc. 4th Berkeley Sympos. Math. Statist. and Prob., vol. II, pp. 593-622. Univ. California Press, Berkeley (1961) 